\title{
Feedback from Mass Outflows in Nearby Active Galactic Nuclei I. UV and X-ray Absorbers
}

\author{
D.M. Crenshaw ${ }^{1}$ and S.B. Kraemer ${ }^{2}$
}

\begin{abstract}
We present an investigation into the impact of feedback from outflowing UV and X-ray absorbers in nearby $(z<0.04)$ AGN. From studies of the kinematics, physical conditions, and variability of the absorbers in the literature, we calculate the possible ranges in total mass outflow rate $\left(\dot{M}_{\text {out }}\right)$ and kinetic luminosity $\left(L_{K E}\right)$ for each AGN, summed over all of its absorbers. These calculations make use of values (or limits) for the radial locations of the absorbers determined from variability, excited-state absorption, and other considerations. From a sample of 10 Seyfert 1 galaxies with detailed photoionization models for their absorbers, we find that 7 have sufficient constraints on the absorber locations to determine $\dot{M}_{\text {out }}$ and $L_{K E}$. For the low-luminosity AGN NGC 4395, these values are low, although we do not have sufficient constraints on the X-ray absorbers to make definitive conclusions. At least 5 of the 6 Seyfert 1s with moderate bolometric luminosities $\left(L_{b o l}=10^{43}-10^{45} \mathrm{ergs} \mathrm{s}^{-1}\right)$ have mass outflow rates that are $10-$ 1000 times the mass accretion rates needed to generate their observed luminosities, indicating that most of the mass outflow originates from outside the inner accretion disk. Three of these (NGC 4051, NGC 3516, and NGC 3783) have $L_{K E}$ in the range $0.5-5 \% L_{b o l}$, which is the range typically required by feedback models for efficient self-regulation of black-hole and galactic bulge growth. At least 2 of the other 3 (NGC 5548, NGC 4151, and NGC 7469) have $L_{K E} \gtrsim 0.1 \% L_{b o l}$, although these values may increase if radial locations can be determined for more of the absorbers. We conclude that the outflowing UV and X-ray absorbers in moderate-luminosity AGN have the potential to deliver significant feedback to their environments.
\end{abstract}

Subject headings: galaxies: active - galaxies: Seyfert - galaxies: kinematics and dynamics

\footnotetext{
${ }^{1}$ Department of Physics and Astronomy, Georgia State University, Astronomy Offices, One Park Place South SE, Suite 700, Atlanta, GA 30303; crenshaw@chara.gsu.edu

${ }^{2}$ Institute for Astrophysics and Computational Sciences, Department of Physics, The Catholic University of America, Washington, DC 20064
} 


\section{Introduction}

Active galactic nuclei (AGN) are fed by accretion of matter onto supermassive black holes (SMBHs), generating huge amounts of radiation from very small volumes. In addition to radiative feedback (e.g., Ciotti, Ostriker, \& Proga 2010), AGN provide feedback via mass outflows of ionized gas into their environments, which are thought to play a critical role in the formation of large-scale structure in the early Universe (e.g., Scannapieco \& Oh 2004; Di Matteo et al. 2005), chemical enrichment of the intergalactic medium (e.g., Khalatyan et al. 2008), and self-regulation of SMBH and galactic bulge growth (e.g., Hopkins et al. 2005). For example, the currently popular explanation for the relation between the SMBH mass and the stellar velocity dispersion in the bulge, the $M_{B H}-\sigma_{*}$ relation (Gebhardt et al. 2000; Ferrarese \& Merritt 2000), is that AGN feedback results in evacuation of gas from the bulge, quenching of star formation, and a halt to the growth of the SMBH and bulge. However, we have very little information on the validity of this explanation, the frequency and magnitude of mass outflows from AGN, or the detailed physical mechanisms of feedback.

Mass outflows from AGN arise in two principal sources: radio jets and "AGN winds". Most feedback models have concentrated on the former, because jets are very powerful and are clearly seen to impact their host galaxies and extragalactic environments. However, jets are narrowly focused, and radio-loud AGN with strong jets occur in only $5-10 \%$ of the AGN population (Rafter et al. 2009, and references therein). Thus, it is important to consider the impact that AGN winds have on their environments. These winds are often revealed through UV and X-ray absorption lines that are blueshifted with respect to their host galaxies, with typical outflow velocities up to $2000 \mathrm{~km} \mathrm{~s}^{-1}$ in Seyfert 1 galaxies (Crenshaw, Kraemer, \& George 2003a) and potentially much higher velocities in quasars (Ganguly \& Brotherton 2008), especially in broad absorption-line (BAL) quasars (with maximum outflow velocities between 3000 and 25,000 $\mathrm{km} \mathrm{s}^{-1}$, Gibson et al. 1999). Winds in another form have also been detected as outflows of emission-line gas in the narrow (emission) line regions (NLRs) of nearby AGN, with outflow velocities up to $\sim 1500 \mathrm{~km} \mathrm{~s}^{-1}$ on scales of hundreds of parsecs (Crenshaw et al. 2010; Fischer et al. 2011).

Although AGN feedback is usually discussed in terms of high-luminosity quasars interacting with their environments at high redshifts, it is useful to explore the impact of winds from nearby AGN at moderate luminosities. In particular, Seyfert 1 galaxies, with bolometric luminosities $\mathrm{L}_{b o l} \lesssim 10^{45} \mathrm{erg} \mathrm{s}^{-1} \mathrm{~cm}^{-2}$, are bright enough in apparent magnitude for high-resolution spectroscopy to study the detailed physics of their winds. This pursuit is im- 
portant for gauging the importance of winds from moderate-luminosity AGN in general, and for understanding the mechanisms of feedback in more luminous AGN at higher redshifts.

In this paper, we concentrate on outflowing UV and X-ray absorbers, seen as kinematic components of blueshifted absorption lines in the spectra of Seyfert 1 galaxies. A large number of high-resolution UV and X-ray spectra suitable for this purpose have been obtained over the past couple of decades with the Hubble Space Telescope (HST), Far Ultraviolet Spectroscopic Explorer (FUSE), Chandra X-ray Observatory (CXO), and X-ray Multi-Mirror Mission (XMM-Newton). The absorbers have been characterized in great detail by ourselves and others with the use of multi-epoch observations and detailed photoionization models. In this paper, we use the published results to estimate the magnitude of feedback from UV and X-ray absorbers, by determining (or placing limits on) the total mass outflow rate $\left(\dot{M}_{\text {out }}\right)$ and kinetic luminosity $\left(L_{K E}\right)$ in individual AGN. In a subsequent paper, we will examine the NLR outflows in Seyfert galaxies and their importance for AGN feedback.

\section{Sample}

In order to quantify the feedback from outflowing absorbers in nearby AGN, we need UV and X-ray spectra at high spectral resolutions to isolate and measure the kinematic components of absorption. We apply this criterion by selecting studies with HST UV observations over the $1150-3200 \AA$ range at velocity resolutions of $7-30 \mathrm{~km} \mathrm{~s}^{-1}$ (FWHM) with the Goddard High-Resolution Spectrograph (GHRS), Space Telescope Imaging Spectrograph (STIS), and Cosmic Origins Spectrograph (COS). We can also use spectra from FUSE in the range 900 - $1200 \AA$ at a resolution of $\sim 15 \mathrm{~km} \mathrm{~s}^{-1}$ (FWHM). To obtain the highest possible spectral resolutions in X-rays, we make use of studies with observations primarily from the grating spectrometers on $C X O$ in conjunction with the HETG $(0.4-10 \mathrm{keV}$ coverage, $\geq 300$ $\mathrm{km} \mathrm{s}^{-1}$ resolution in the regions of interest) or LETG $\left(0.1-3 \mathrm{keV}\right.$ coverage, $\geq 150 \mathrm{~km} \mathrm{~s}^{-1}$ resolution) gratings, and from the $X M M-N e w t o n$ Reflection Grating Spectrometer (RGS) $\left(0.25-2.5 \mathrm{keV}\right.$ coverage, $\geq 400 \mathrm{~km} \mathrm{~s}^{-1}$ resolution).

Our second criterion is that there must be detailed photoionization models for both UV and X-ray absorbers in each AGN in the literature, so that we have the measurements needed for feedback determinations. The models are based on measured ionic column densities and provide the ionization parameters and hydrogen column densities that characterize the physical conditions in the gas. In the UV, one must correct for partial covering of the background emission (continuum, broad line, etc.) by the absorbers to avoid underestimating the column densities (Arav et al. 2002, 2003; Crenshaw et al. 2003a). 
We use the dimensionless ionization parameter $U$, which is the density of photons with energies $\geq 13.6 \mathrm{eV}$ divided by the number density of hydrogen atoms at the illuminated face of the slab:

$$
\mathrm{U}=\int_{\nu_{0}}^{\infty} \frac{\mathrm{L}_{\nu} h \nu}{4 \pi r^{2} n_{H} c} d \nu
$$

In studies where the ionization parameter $\xi\left(=L_{i o n} / n_{H} r^{2}\right)$ is given, we use the conversion $\log (U)=\log (\xi)-1.5$ based on a typical Seyfert 1 spectral energy distribution (SED), which we take to be several joined power laws of the form $F_{\nu} \propto \nu^{-\alpha}$, with $\alpha=1$ below $13.6 \mathrm{eV}$, $\alpha=1.4$ over the range $13.6 \mathrm{eV}<\mathrm{h} \nu<1000 \mathrm{eV}$, and $\alpha=0.7$ above $1000 \mathrm{eV}$ (Kraemer et al. 2001).

The other model parameter that we use is the total hydrogen density $N_{H}=N_{H I}+N_{H I I}$ in units of $\mathrm{cm}^{-2}$. $N_{H}$ is sensitive to the elemental abundances used in the photoionization models, which are specified by the studies that present these models. In most cases, solar abundances (Asplund et al. 2009) are used, but there are a few notable exceptions of higher metallicity outflows (see Arav et al. 2007; Fields et al. 2007).

Our third criterion is that we restrict our sample to apparently bright AGN with broad emission lines at redshifts $z<0.04$ that are suitable for high resolution spectroscopy. We do not include ultrafast outflows (with outflow velocities $>10,000 \mathrm{~km} \mathrm{~s}^{-1}$, Tombesi et al. 2010), as the nature and global covering factor of these absorber outflows are not well understood. We note that neither BALs (e.g., Gibson et al. 2009) nor very high velocity UV absorbers (e.g., Hamann et al. 2011) have been detected in AGN at these low redshifts.

The above criteria result in a sample of 10 nearby Seyfert 1 galaxies listed in Table 1. We note that NGC 4051 and Akn 564 are often classified as narrow-line Seyfert 1 galaxies, with FWHM(broad $\mathrm{H} \beta)<2000 \mathrm{~km} \mathrm{~s}^{-1}$, and NGC 4395 is often called a "dwarf Seyfert 1" due to its low luminosity.

In Table 1, we give some of the fundamental parameters for each AGN in our sample. From the literaure, we give the estimated radii of the broad line regions (BLRs) in light days from reverberation mapping of the broad $\mathrm{C} I V$ and $\mathrm{H} \beta$ emissions, $r_{C I V}$ and $r_{H \beta}$ respectively (Peterson et al. 2004). For each AGN, we also list the derived mass of the SMBH, $M_{B H}$, and the monochromatic luminosity at $5100 \AA, \lambda \mathrm{L}_{\lambda}(5100)$, both obtained from the listed reference. In addition to $M_{B H}$, we give the bolometric luminosity $\mathrm{L}_{b o l}=9.8 \lambda \mathrm{L}_{\lambda}(5100)$ (McLure \& Dunlop 2004), Eddington ratio $L_{b o l} / L_{E d d}$, and mass accretion rate needed to generate the observed luminosity $\dot{M}_{a c c}=L_{b o l} / \eta c^{2}$, where we assume $\eta=0.1$ (Peterson et al. 1997). 


\section{Analysis}

\subsection{Feedback Calculations}

To determine the mass outflow rate for each absorber, we use the equation

$$
\dot{M}_{\text {out }}=4 \pi r N_{H} \mu m_{p} C_{g} v_{r}
$$

(Crenshaw et al. 2003a), where $r$ is the absorber's radial location (i.e., its distance from the central SMBH), $N_{H}$ is the hydrogen column density, $\mu$ is the mean atomic mass per proton (=1.4 for solar abundances), $m_{p}$ is the proton mass, $C_{g}$ is the global covering factor $(=0.5)$, and $v_{r}$ is the radial velocity centroid. The kinetic luminosity is then:

$$
L_{K}=1 / 2 \dot{M}_{\text {out }} v_{r}^{2}=2 \pi r N_{H} \mu m_{p} C_{g} v_{r}^{3}
$$

The average global covering factor can be determined statistically from $C_{g}=C_{\text {los }} f$, where $C_{l o s}$ is the average covering factor of the background emission in the line of sight and $f$ is the fraction of AGN that show intrinsic absorption (Crenshaw et al. 2003a). A number of studies have shown that $C_{g} \approx 0.5$ for both UV (Crenshaw et al. 1999; Dunn et al. 2007) and X-ray absorbers (Reynolds 1997; George et al. 1998; Winter 2010). Technically, this factor could be lower by a factor of 2 to 3, because, according to unified models, we see Seyfert 1 s over a restricted range of viewing angles. On the other hand, if it turns out that half of Seyfert 1s are completely covered and the other half are not covered at all, we could increase $C_{g}$ by a factor of $\sim 2$ for the former. Thus, we keep $C_{g}=0.5$, with the understanding that this value could be off by a factor of $\sim 2$ either way.

\subsection{Absorber Distances}

In order to determine $\dot{M}_{\text {out }}$ and $L_{K E}$ for each absorber, we do not assume continuous, radiatively driven outflows at constant velocity (e.g., Blustin et al. 2005), as there is no evidence that this assumption is valid. Thus, we must know the radial location $r$ of the absorber, which is the most difficult parameter to determine in the above equations. Fortunately, we can use absorption from excited states or absorption variability to determine (or place limits on) $r$. As discussed in Crenshaw et al. (2003a), column densities of excited levels populated by collisional excitation can be used to determine the electron number density $\mathrm{n}_{e}$ (and hence the hydrogen number density $\mathrm{n}_{H}$ ). If the absorption responds to a decrease in ionizing flux, one can also determine $\mathrm{n}_{e}$ and $\mathrm{n}_{H}$ from the recombination time scale (Nicastro et al. 1999). Photoionization models of the ionic column densities provide $U$, and together 
with $n_{H}$ and the ionizing luminosity in photons, the radial location $r$ via equation 1 . If the absorption responds to an increase in ionizing flux, $r$ can be determined directly from the ionization time scale (Crenshaw et al. 2003a), which depends on the ionizing flux incident on the cloud. In many cases, we only have upper limits on the time scale over which the absorption lines vary, which yield lower limits on $\mathrm{n}_{H}$ and upper limits on $r$. On the other hand, if the absorbers do not respond to large changes in the ionizing continuum, we can obtain upper limits to $\mathrm{n}_{H}$ and lower limits to $r$. The dominant uncertainites in these values come from uncertainties in the photoionization model parameters; typical uncertainities are $\sim 0.3$ in $\log (r)$ (Crenshaw et al. 2003, 2009; Kraemer et al. 2006).

We also use other clues to determine limits on $r$ for the absorbers. The size of the BLR responsible for most of the broad $\mathrm{C} I V$ emission, $r_{C I V}$, serves as the absolute minimum for the radial location of the absorber, because in nearly every case it has been shown that the depth of the C IV absorption exceeds the continuum flux, and thus this region must be at least partially covered (Crenshaw et al. 2003a). As shown in Table 1, only 4 AGN in our sample have direct determinations of $r_{C I V}$ via reverberation mapping (Peterson et al. 2004, 2005). They all have determinations of the size of the region responsible for broad $\mathrm{H} \beta$ emission, $r_{H \beta}$, which is known to be larger than $r_{C I V}$ (Peterson et al. 2004, Vestergaard \& Peterson 2006). For the AGN in Table $1, r_{H \beta} / r_{C I V}=1.8-2.7$, whereas Netzer (2009) suggests that this ratio in general is $\sim 3$, based on scaling relations. To be conservative, for the AGN without direct determinations of $r_{C I V}$ we use $r_{C I V}=r_{H \beta} / 3.0$ as the absolute minimum for the radial location of the absorber. Uncertainites in the BLR sizes from reverberation mapping are 0.1 to 0.2 in $\log (r)$ (Peterson et al. 2004). We note that the BLR size does not provide a constraint on the X-ray absorbers, which lack significant underlying broad-line emission.

Limits on $r$ can also be determined if there is evidence for or against the absorber covering the NLR (Crenshaw et al. 2002, 2009), as long as there is an estimate of the NLR size. In some cases, the relative locations of the absorbers can be deduced from the photoionization models by finding, for example, that one absorber cannot be shielded by another, and therefore must be inside of the latter (Kraemer et al. 2002).

We can determine an absolute maximum for the radial location of an absorber, based on the requirement that the thickness of the absorber cannot exceed its distance from the SMBH:

$$
r \leq \frac{L_{i o n}}{N_{H} \xi}
$$

(Blustin et al. 2005), where $L_{i o n}$ is the ionizing luminosity (27\% of $\mathrm{L}_{b o l}$ for our SED) and $\xi$ is determined from $U$ as previously discussed. Uncertainties in this limit once again come from those in $U$ and $N_{H}$, and are on the order of 0.3 in $\log (r)$. This upper limit and the lower limit from the BLR size provide extreme ranges to the absorber radial locations that 
are only occasionally useful. Much tighter constraints come from absorption from excited levels or variable absorption.

\section{Results}

We give a detailed account of the measurements from the literature that we adopted for each AGN in the Appendix. We list these measurements and the derived minimum and maximum $r, \dot{M}_{\text {out }}$, and $L_{K E}$ for each absorber in Table 2, when these could be determined. Here we take a graphical look at some of these values.

\subsection{Absorption Measurements}

In Figure 1, we show the full width at half-minimum (FWHM) of each absorption component versus the radial-velocity centroid for the UV absorbers (as explained in the Appendix, we do not have reliable FWHM for the X-ray absorbers). There is no apparent correlation between the two parameters. The absorbers in these Seyfert 1s span the velocity range -2000 to $+200 \mathrm{~km} \mathrm{~s}^{-1}$ with respect to the systemic velocity of the host galaxy (with the exception of two X-ray absorbers given in the Appendix) and the FWHM range is 20 to $500 \mathrm{~km} \mathrm{~s}^{-1}$ (with one exception).

In Figure 2, we plot $\log (U)$ versus $v_{r}$ for both UV and X-ray absorbers. The X-ray absorbers tend to have higher $U$, as expected. Again, there is no correlation, despite a few claims in the literature of trends in individual AGN. There is a huge range in ionization parameter: $\log (U) \approx-2$ to 4 .

In Figure 3, there appears to be a positive correlation between $\log (U)$ and $\log \left(N_{H}\right)$. The lack of high-ionization columns at low column densities can be explained by the sensitivity limits of current X-ray missions. It is not clear why there are no large-column, low-ionization components detected in the UV - this may represent a real physical constraint. There is a gap around $\log (U)=0$ in this plot, also seen in Figure 2. This gap may represent the manner in which absorbers are typically identified, which is from the presence of $\mathrm{C} I V$ absorption in the UV, and O IV or O VIII absorption in the X-rays, and could potentially be filled in with photoionization models of AGN observed by FUSE, which provide access to O VI at low redshift. To test this notion we ran a photoionization model with $\log (U)=0, \log \left(N_{H}\right)$ $=21.0$, and the above SED, and found $\mathrm{N}(\mathrm{C}$ IV $)=3.8 \times 10^{13} \mathrm{~cm}^{-2}$, which can be difficult to detect in HST spectra (Crenshaw et al. 1999), and $\mathrm{N}(\mathrm{O} \mathrm{VI})=1.2 \times 10^{16} \mathrm{~cm}^{-2}$, which

would be strong in FUSE spectra (Dunn et al. 2007). Note that the extreme point in Figure 
3 at $\log (U)=-0.39, \log \left(N_{H}\right)=22.93$ is the "D+Ea" component in NGC 4151 (see Table 3), which may result from a special line of sight near the edge of the NLR bicone (Crenshaw \& Kraemer 2007).

We plot the values or limits for the radial location $r$ (in pc) along with $v_{r}$ for each absorber in Figure 4. There is no apparent correlation between the two. Compared to the UV absorbers, the X-ray absorbers tend to be concentrated toward smaller $r$, but there is no preference in terms of $v_{r}$. The main result from this figure is that the vast majority of UV and X-ray absorbers lie between 0.01 and 100 pc from the central SMBH, outside of the BLR and inside much of the classic NLR (i.e, in the inner NLR [Crenshaw \& Kraemer 2005] or the "intermediate-line region" [Crenshaw \& Kraemer 2007; Crenshaw et al. 2009]). The issue of where the absorbers actually originate, in contrast to where they are currently located, is discussed in the next subsection and Section 5.

\section{2. $\quad$ Feedback Parameters}

In Table 2 we give the range in feedback parameters $\dot{M}_{\text {out }}$ and $L_{K E}$ for each AGN from the minimum and maximum values summed over all absorbers. We also give the ratio of outflow to accretion rate $\dot{M}_{\text {out }} / \dot{M}_{a c c}$ and the ratio of kinetic to bolometric luminosity $L_{K E} / L_{b o l}$ for each AGN. As discussed in the Appendix, we were unable to obtain reliable limits for Mrk 279, Mrk 509, and Akn 564, due primarily to the lack of constraints on radial locations for most of their absorbers. Thus, we have feedback values for 7 of the 10 Seyfert 1 galaxies in our original sample.

We plot the range in $\log \left(\dot{M}_{\text {out }} / \dot{M}_{\text {acc }}\right)$ against $L_{b o l}$ in Figure 5 . For 5 of the 7 Seyfert 1 galaxies in our sample, the mass outflow rate exceeds the mass accretion rate by a factor of 10 to 1000 (NGC 7469 provides only an upper limit and NGC 4395 is a low-luminosity Seyfert). Thus, the vast majority of this type of outflow in moderate-luminosity AGN must originate outside of the inner accretion disk, where most of the AGN's luminosity is generated; otherwise, the inner accretion disk would likely quickly dissipate. There may be a slight correlation of $\dot{M}_{\text {out }} / \dot{M}_{a c c}$ with $L_{b o l}$, but better constraints and more data are needed, especially at $L_{b o l}=10^{41}-10^{43} \mathrm{ergs} \mathrm{s}^{-1}$, to test for a trend. It is possible that $\dot{M}_{\text {out }}$ is indeed very low for NGC 4395, but this value does not include contributions from the X-ray absorbers (see the Appendix).

In Figure 6, we plot $\log \left(L_{K E} / L_{b o l}\right)$ against $L_{b o l}$. Out of the six moderate-luminosity AGN (i.e., excluding NGC 4395), three (NGC 4051, NGC 3516, and NGC 3783) have kinetic luminosities that are approximately $0.5 \%$ to $5 \%$ of their bolometric luminosities, which is the 
range typically assumed by feedback models (Hopkins \& Elvis 2010, and references therein). NGC 5548 could potentially be in this range, whereas NGC 4151 and NGC 7469 are at the $\lesssim 0.1 \%$ level. Once again, $L_{K E} / L_{b o l}$ appears to be low for the dwarf Seyfert 1 galaxy NGC 4395, but the values for the X-ray absorbers need to be included to test this possibility. Excluding NGC 4395, we see no clear trend in $\dot{M}_{\text {out }} / \dot{M}_{a c c}$ or $L_{K E} / L_{b o l}$ with either $L_{b o l} / L_{E d d}$ or black hole mass. However, the current sample is small, and detailed studies of more AGN outflows are needed to explore the dependence of feedback on fundamental AGN properties.

\section{Conclusions and Discussion}

The total mass outflow rates from UV and X-ray absorbers in the moderate-luminosity AGN in our sample are typically 10 - 1000 times the accretion rates needed to provide the observed luminosities. The majority of this outflow must therefore originate from outside the inner accretion disk. There are two interesting possibilities. One is that a large reservoir of gas has accumulated, in a torus or other circumnuclear structure, and the gas is being accelerated off this structure (e.g., Krolik \& Kriss 2001). A related possibility is that the gas is continuously accelerated directly off the fueling flow over a range of distances. There is some evidence for the latter in larger scale NLR outflows (Crenshaw et al. 2010; Fischer et al. 2010).

Previous AGN feedback models have typically required that $\sim 5 \%$ of the bolometric luminosity of an AGN be converted into kinetic luminosity in order to regulate the growth of a SMBH and its galactic bulge (Di Matteo et al. 2005; Hopkins et al. 2005). However, Hopkins $\&$ Elvis (2010) have presented a model in which only $\sim 0.5 \%$ conversion is required. We find that the total kinetic luminosity, summed over all absorbers, is $0.5 \%$ to $5 \%$ of the bolometric luminosity for half of our moderate-luminosity AGN, in the range required by the models. Of the remaining three, 2 of these have $L_{K E} \gtrsim 0.1 \% L_{b o l}$ and one has $L_{K E} \lesssim 0.1 \% L_{b o l}$. However, we emphasize that some absorbers still have no usable limits on $r$, so that future work may actually increase these values of $\dot{M}_{\text {out }}$ and $L_{K E}$. NLR outflows, which we consider in a subsequent paper, will further increase these values. Thus, we find that the outflowing UV and X-ray absorbers in moderate-luminosity $\left(10^{43}-10^{45} \mathrm{ergs} \mathrm{sec}^{-1}\right)$ AGN have the potential to deliver significant feedback to their environments.

This research has made use of the NASA/IPAC Extragalactic Database (NED) which is operated by the Jet Propulsion Laboratory, California Institute of Technology, under contract with the National Aeronautics and Space Administration. This research has made use of NASA's Astrophysics Data System Bibliographic Services. 


\section{A. Details on Outflowing Absorbers in Individual AGN}

In Table 3, we give detailed measurements of individual absorption components from the literature and the corresponding references in the subsection for each AGN . The absorption component names are from the original studies. We give $v_{r}$ for each UV and X-ray component, and the FWHM for each UV component only. In cases where FWHM values were available for multiple lines, we chose those values that corresponded to less saturated lines in order to minimize the effects of saturation on broadening the observed profiles. The X-ray components tend to be unresolved or barely resolved in $C X O$ or XMM-Newton grating spectra (Kaspi et al. 2003), and even the resolved profiles are often likely blends of several distinct kinematic components (Gabel et al. 2003). Furthermore, many of the quoted values are from the velocity dispersion used in the photoionization model to fit the spectrum, and not from direct measurements. Thus, we do not include FWHM values for the X-ray absorbers in our compilation.

For each component in Table 3 with an available photoionization model, we give $\log (U)$ and $\log \left(\mathrm{N}_{H}\right)$. For studies where the ionization parameter $\xi$ was given, we converted to $\log (U)$ as described in Section 2. If there were different values at different epochs due to variability, we averaged those values to get the $\log (U)$ and $\log \left(N_{H}\right)$ in the table. For each UV absorber, we give the size of the C IV BLR $r_{C I V}$ from Table 1 as the absolute minimum values for $r$. We also give the absolute maximum value for $r, r_{>\Delta r}$. In most cases, however, we do not use this value, because $r_{>\Delta r}$ is on the order of kpcs or more. However, it is useful for a few X-ray absorbers.

The most crucial parameters for this study are our adopted $r_{\min }$ and $r_{\max }$ in Table 3, which are the lower and upper limits for the radial locations of the absorbers. In most cases, we were able to use values from the literature based on variability of the absorption (or lack thereof) and/or absorption (or its absence) from excited levels to provide density limits, which, combined with $U$, yield limits to the radial locations. In a few specific cases (e.g., in NGC 3783 and NGC 4151), the actual values have been determined, and these are listed as both $r_{\min }$ and $r_{\max }$. When these values are not available, we resort to $r_{C I V}$ and $r_{>\Delta r}$ when appropriate.

Finally, we give minimum and maximum values for the feedback parameters $\dot{M}_{\text {out }}$ and $L_{K E}$ in Table 3, based on the radial location limits. We then sum the contributions from the UV and X-ray absorbers to obtain limits for the total $\dot{M}_{\text {out }}$ and $L_{K E}$ in each AGN for use in Table 2. In a few cases, particular UV and X-ray absorbers have been shown to arise in the same gas, and we do not count both when determining the totals. If a particular component has no maximum value, we use its minimum value when determining a value for the total

maximum $\dot{M}_{\text {out }}$ and $L_{K E}$. We give a detailed discussion of these determinations for each 
AGN in the following subsections.

\section{A.1. NGC 3516}

NGC 3516 shows eight kinematic components of intrinsic absorption in the UV (Component 1 has two physical subcomponents, $1 \mathrm{a}$ and $1 \mathrm{~b}$, with different $U$ and $N_{H}$; Kraemer et al. 2002) and at least three distinct components in X-rays (Turner et al. 2005, 2008, 2011). Based on detailed photoionization models and shielding of the ionizing continuum by these components, Kraemer et al. showed that the UV components follow the sequence $3+4$ (blended at most epochs), 2, 1 , and 5-8 in order of increasing radial distance from the central SMBH, and Components $1-4$ are responsible for the X-ray absorption identified by Netzer et al. (2002). We therefore do not use this X-ray component, labeled "UV" in Turner et al. (2005), in determining the total mass outflow rate and kinetic luminosity. Lower limits to the radial distances of Components $1-4$ were determined from the lack of metastable C III absorption by Kraemer et al. (2002). As shown in Table 3, we have increased these limits to account for the lack of C III absorption at levels populated by lower densities than

originally considered (specifically, the transition from $\mathrm{J}=0$; see Gabel et al. 2005). The lower limit for Component 1 can also be applied to UV components 5 - 8, but no reasonable upper limits are available for these components. An upper limit to the radial location of "UV" of $\sim 0.4 \mathrm{pc}$ was determined from the variability of O VII absorption in the X-rays (Netzer et al. 2002; Kraemer et al. 2002). This upper limit is smaller than the lower limits for UV components 1 and 2, but not 3+4, suggesting that it was the latter UV component that varied (consistent with its previous strong variability; Kraemer et al. 2002).

The ionizing radiation for the other two X-ray components in Turner et al. (2005), "Hi" and "Heavy", cannot be shielded by "UV" (Kraemer et al. 2002), and they must therefore lie inside of the latter, providing an upper limit to their radial distances. The $\Delta r / r<1$ requirement puts a tighter constraint on X-ray component Heavy - it must be lie at $r \leq 0.11$ pc. Heavy shows partial covering in the line of sight and possibly higher columns than listed in Table 3 at some epochs (Turner et al. 2008; 2011), so its upper limits for mass outflow rates and kinetic luminosities are approximate. Upper limits are not available for most of the UV components, so the maximum values for $\dot{M}_{\text {out }}$ and $L_{K E}$ in NGC 3516 could be higher. 


\section{A.2. NGC 3783}

NGC 3783 has three distinct kinematic components of intrinsic UV absorption (Kraemer et al. 2001; Gabel et al. 2005) and three components of X-ray absorption (Netzer et al. 2003). UV component 1 consists of two physical subcomponents (1a and 1b); we assume these are co-located because they are at the same velocity. Metastable C III absorption provides the density and distance of UV 1b. Monitoring of the strong absorption variations in UV components 2 and 3 provides upper limits to their distances (Gabel et al. 2005) and absorption of the BLR provides lower limits. X-ray component XLI has similar $U$ and $v_{r}$ coverage as components $1 \mathrm{~b}, 2$, and 3 , suggesting it arises in the same gas, but it has $\sim 3$ times the $\mathrm{N}_{H}$ of the UV absorbers (Gabel et al. 2005). We therefore exclude XLI in our lower limits, but include its remaining column, after subtracting the UV columns in our upper limits, for outflow rates and kinetic luminosities. The lack of variability in the X-ray components on a time scale of $\sim 10$ days, despite strong continuum variations, provides lower limits to the radial locations of X-ray components XMI and XHI (Netzer et al. 2003). We use the $\Delta \mathrm{r} / \mathrm{r}$ constraints for upper limits to the radial locations of XMI and XHI, and set the upper limit of XLI to that of the most distant UV component (3), as discussed above. Krongold et al. (2005) find an upper limit of $\sim 6$ pc for this lower-ionization component, which is consistent with the other determinations.

\section{A.3. NGC 4051}

NGC 4051 shows 9 distinct components of UV absorption in its STIS (Collinge et al. 2001) and COS (Kraemer et al. 2012) spectra, in addition to a component that is clearly due to the ISM in our Galaxy. Component 1 likely arises in our Galaxy and Components 8 and 9 (plus Collinge et al.'s Component 10) likely arise in the host galaxy of NGC 4051. Components 3, 4, and 6 are weak and difficult to separate from the stronger Components 2, 5 , and 7. The latter provide the bulk of the outflow in the UV and are modeled by Kraemer et al. (2012). Steenbrugge et al. (2009) use 4 zones to model the X-ray absorption, similar to those in Lobban et al. (2011). We use Steenbrugge et al.'s components because these authors provide distance constraints. Based on photoionization parameters and velocity correspondences, Kraemer et al. (2012) find that Steenbrugge et al.'s X-ray 1 and 2 are likely the same as UV 7 and 5, respectively, and thus we do not include these UV components in our totals for mass outflow rates and kinetic luminosities. Steenbrugge et al.'s X-ray 3 is at the same approximate velocity as UV 2, suggesting that they are co-located, but their $U$ and

$N_{H}$ are very different. We therefore include both in our calculations. Based on the lack of variability in X-ray 1, 3, and 4, Steenbrugge et al. obtained lower limits to their locations. 
The $r>\Delta r$ requirement for Component 4 gives an upper limit consistent with the above lower limit to within the uncertainty of $\sim 0.3$ dex.Component 2 varied over this time period, providing an upper limit to its radial location.

Steenbrugge et al.'s Component 4 has unusually high $v_{r}, U$, and $N_{H}$, and it dominates the mass outflow parameters. However, it does not qualify as an ultrafast outflow (Tombesi

et al. 2010). Thus, the global covering factor of this component is unclear. We therefore use $C_{g}=0.5$ for the upper limits to its $\dot{M}_{\text {out }}$ and $L_{K E}$, but scale these numbers by a factor of 0.1 (i.e., $C_{g}=0.05$ ) for the lower limits to account for its uniqueness among our sample of 10 Seyfert 1 galaxies.

\section{A.4. NGC 4151}

NGC 4151 has many components of UV absorption that were first identified by Weymann et al. (1997). Their D and E components are not separable and are lumped together as a single kinematic component "D+E" in subsequent studies (e.g., Crenshaw et al. 2000; Kraemer et al. 2001). Components $\mathrm{D}+\mathrm{E}$ and $\mathrm{E}^{\prime}$ are modeled as four (a, b, c, d) and two (a, b) physical subcomponents, respectively, in Kraemer et al. (2005, 2006), who found that subcomponent D+Ea is responsible for the low-ionization X-ray absorption. Another component ("Xhigh") is needed to explain the high-ionization absorption lines in the X-ray region of the spectrum. Distances to all of the UV absorbers are obtained from absorption lines arising from metastable and/or fine-structure excited levels. All D+E subcomponents are assumed to lie at the same distance as $\mathrm{D}+\mathrm{Ea}(0.1 \mathrm{pc})$ due to their velocity correspondence. Component $\mathrm{D}^{\prime}$ is screened by $\mathrm{D}+\mathrm{Ea}$ and must lie outside of it. Xhigh has the same approximate radial velocity of $\mathrm{D}+\mathrm{Ea}$ and must lie inside of the latter, because photoionization models demonstrate that Xhigh is not shielded from the ionizing radiation by $\mathrm{D}+\mathrm{Ea}$ (Kraemer et al. 2005). UV components $\mathrm{A}$ and $\mathrm{C}$ are in the NLR at large distances from the central SMBH, and we include these only in the upper limits for mass outflow rates and kinetic luminosities, even though their distances are known, because they may not have global covering factors as large as 0.5.

\section{A.5. NGC 4395}

NGC 4395 is a nearby dwarf Seyfert 1 (Filippenko \& Sargent 1989), with a very low luminosity $\left(\mathrm{L}_{b o l} \approx 5 \times 10^{40} \mathrm{ergs} \mathrm{s}^{-1}\right)$ and black-hole mass $\left(\mathrm{M} \approx 3.6 \times 10^{5} \mathrm{M}_{\odot}\right)$ (Peterson et al. 2005). Nevertheless, its UV spectrum shows two UV absorbers that are outflowing from 
its nucleus (Crenshaw et al. 2004, and references therein). Baskin \& Laor (2008) determined the physical conditions in the two absorbers and pointed out that they are likely between its tiny C IV BLR $(\sim 0.4$ light days, Peterson et al. 2004$)$ and inner NLR $\left(\sim 2.3 \times 10^{-2}\right.$ pc, Kraemer et al. 1999). Moreover, NGC 4395 shows strong evidence for warm absorbers in its X-ray spectra (Iwasawa et al. 2000; Shih et al. 2003; Moran et al. 2005). We give Shih et al.'s characterization of a constant and a variable zone of ionized absorption in the Xrays. Unfortunately, we are unable to include feedback parameters for the X-ray absorbers, because there has been no determination of their radial velocities.

\section{A.6. Mrk 279}

Mrk 279 has five principal components of UV absorption; Component 1 likely arises in the host galaxy and the low ionization lines of Component 4 may arise in its halo or a companion galaxy (Scott et al. 2004, 2009; Gabel et al. 2005). Arav et al. (2007) used

photoionization models to determine the physical conditions in Component 2. Scott et al. (2009) find that variability of the UV absorption lines is due to both varying contributions from emission regions that have different covering factors and intrinsic variation, and the locations of the absorbers have not been determined. Ebrero et al. (2010) find two warm absorbers in X-ray spectra (see also Constantini et al. 2007). X-ray component 1 and UV 2 have similar $v_{r}$ and $U$, but the former has $\sim 10$ times higher $N_{H}$. Ebrero et al. find no strong evidence for or against variability in the X-ray absorbers, and thus no distances or feedback parameters are available for this AGN.

\section{A.7. NGC 5548}

NGC 5548 shows five principal components of intrinsic UV absorption (Crenshaw et al. 1999, 2003b; Mathur et al. 1999). None of the components show evidence for strong variability in $U$ despite large-scale continuum changes, indicating distances $>70 \mathrm{pc}$ from the central SMBH (Crenshaw et al. 2009). However, a large portion of Component 3 is responsible for some of the X-ray absorption described in Steenbrugge et al. (2005), which Detmers et al. (2008) place at a distance $<7$ pc from the SMBH, based on ionization changes. Andrade-Velázquez et al. (2010) find four components of X-ray absorption: high-velocity super-high ionization phase (HV-SHIP), high-velocity high-ionization phase (HV-HIP), lowvelocity high-ionization phase (LV-HIP), and low-velocity low-ionization phase (LV-LIP). Krongold et al. (2010) find no response of HV-SHIP to changes in the ionizing continuum, and we assume that HV-HIP is co-located, putting the HV components at distances $>0.03$ 
pc from the SMBH. The two LV components are very similar to UV Component 3 in radial velocity, average $U$, and total $N_{H}$, so we do not use these for the total $\dot{M}_{\text {out }}$ and $L_{K E}$. Krongold et al. find a possible response of the LV-LIP component, and assuming LV-HIP is co-located, these components are at distances $<3 \mathrm{pc}$, similar to the limit found by Detmers et al (2008). We have no upper limits for components other than UV 3 (LV-HIP+LV-LIP), and we therefore do not include upper limits for total $\dot{M}_{\text {out }}$ and $L_{K E}$.

\section{A.8. Mrk 509}

Mrk 509 has at least seven components of UV absorption (Kriss et al. 2000; Kraemer et al. 2003; Kriss et al. 2011), depending on how the absorption structure is subdivided. Kraemer et al. (2003) give $U$ and $N_{H}$ for the UV components. Kriss et al. (2011) find that Components $1-3$ arise in outflows, whereas Components $4-7$ are close to systemic or at positive velocities, indicating origins in the host galaxy, halo, or other regions not associated with the outflows. Kriss et al. (2011) find variability in UV 1, indicating a radial location $<250$ pc from the central SMBH. Although the UV and X-ray absorbers have the same radial velocity coverage, the latter have higher $U$ and $N_{H}$ (Kriss et al. 2011). Ebrero et al. (2011) give the physical conditions for the three X-ray absorbers in Mrk 509, which are similar to those found by Detmers et al. (2010, 2011). Detmers et al. use mild variability in the highest ionization component (X-ray 3 in the table) to place it at a distance $<0.5 \mathrm{pc}$ from the SMBH. Because we have distances for only one UV and one X-ray absorber, we do not include Mrk 509 in our determination of total $\dot{M}_{\text {out }}$ and $L_{K E}$.

\section{A.9. Akn 564}

Akn 564 shows strong UV absorption lines from a "lukewarm" absorber that reddens the NLR in this NLS1 (Crenshaw et al. 2002), placing it at a distance $>95$ pc from the central SMBH. The absorber has a radial velocity of $-190 \mathrm{~km} \mathrm{~s}^{-1}$, resulting in significant lower limits to $\dot{M}_{\text {out }}$ and $L_{K E}$, assuming a global covering factor of $C_{g}=0.5$. However, we find that $\mathrm{C}_{g} \leq 0.05$ for this absorber; otherwise its emission-lines fluxes for the higher ionization lines would exceed those observed in the NLR (Crenshaw et al. 2002). Thus, the lower limits for $\dot{M}_{\text {out }}$ and $L_{K E}$ should probably be divided by 10 . Smith et al. (2008) find 3 warm absorbers in X-ray spectra of Akn 564, and two of these could produce at least some of the UV absorption (Matsumoto et al. 2004) . There are no reliable distances for the X-ray absorbers, and, given the above concerns about the UV absorber, we do not include this AGN in our determinations of $\dot{M}_{\text {out }}$ and $L_{K E}$. 


\section{A.10. NGC 7469}

The UV spectrum of NGC 7469 has two main kinematic components (Kriss et al. 2000, 2003) that have been modeled by Scott et al. (2005). Scott et al. detect variability of the H I column density in both components, yielding upper limits to their distances. Blustin et al. (2007) give details on three X-ray warm absorbers in NGC 7469. X-ray component 1 is close to UV 2 in both $v_{r}$ and $U$, but has a higher $N_{H}$; nevertheless, the photoionization model for X-ray 1 predicts UV ionic columns that are reasonably good matches to the observed values (Blustin et al. 2007). X-ray components 2 and 3 are similar to UV 1 in $v_{r}$, but have much higher $U$ and $N_{H}$. There is no information on variability of the X-rays absorbers, but reasonable upper limits can be derived for components 2 and 3 from the $\Delta \mathrm{r} / \mathrm{r}$ constraint. We include the resulting upper limits for $\dot{M}_{\text {out }}$ and $L_{K E}$ in our overall feedback determinations. 


\section{REFERENCES}

Andrade-Velázquez, M., et al. 2010, ApJ, 711, 888

Arav, N., Korista, K.T., \& de Kool,, M. 2002, ApJ, 566, 699

Arav, N., et al. 2003, ApJ, 590, 174

Arav, N., et al. 2007, ApJ, 658, 829

Asplund, M., Grevesse, N., Sauval, A.J., \& Scott, P. 2009, ARA\&A, 47, 481

Bentz, M.C., et al. 2006, ApJ, 651, 775

Blustin, A.J., Page, M.J., Fuerst, S.V., Braduardi-Raymont, G., \& Ashton, C.E. 2005, A\&A, 431, 111

Blustin, A.J., et al. 2007, A\&A, 466, 107

Botte, V., Ciroi, S., Rafanelli, P., \& di Mille, F. 2004, AJ, 127, 3168

Ciotti, L., Ostriker, J.P., \& Proga, D. 2010, ApJ, 717, 708

Collinge, M.J., et al. 2001, ApJ, 557, 2

Costantini, E., et al. 2007, A\&A, 461, 121

Crenshaw, D.M., \& Kraemer, S.B. 1999, ApJ, 521, 572

Crenshaw, D.M., \& Kraemer, S.B. 2005, ApJ, 625, 680

Crenshaw, D.M., \& Kraemer, S.B. 2007, ApJ, 659, 250

Crenshaw, D.M., Kraemer, S.B., \& George, I.M. 2003a, ARA\&A, 41, 117

Crenshaw, D.M., et al. 1999, ApJ, 516, 750.

Crenshaw, D.M., et al. 2000, ApJ, 545, L27

Crenshaw, D.M., et al. 2002, ApJ, 566, 187.

Crenshaw, D.M., et al. 2003b, ApJ, 594, 116

Crenshaw, D.M., et al. 2004, ApJ, 612, 152

Crenshaw, D.M., et al. 2009, ApJ, 698, 291

Crenshaw, D.M., et al. 2010, AJ, 139, 871

Denney, K.D., et al. 2010, ApJ, 721, 715

Detmers, R.G., Kaastra, J.S., Costantini, E., McHardy, I.M., \& Verbunt, F. 2008, A\&A, 488,67

Detmers, R.G., et al. 2010, A\&A, 516, 61 
Detmers, R.G., et al. 2011, A\&A, 534, 38

Di Matteo, T., Springel, V., \& Hernquist, L. 2005, Nature, 433, 604

Dunn, J.P., Crenshaw, D.M., Kraemer, S.B., \& Gabel, J.R. 2007, AJ, 134, 1061

Ebrero, J., et al. 2010, A\&A, 520, 36

Ebrero, J., et al. 2011, A\&A, 534, 40

Ferrarese, L. \& Merritt, D. 2000 ApJ, 539, 9

Fields, D.L., Mathur, S., Krongold, Y., Williams, R., \& Nicastro, F. 2007, ApJ, 666, 828

Filippenko, A.V., \& Sargent, W.L.W. 1989, ApJ, 342, L11

Fischer, T.C., Crenshaw, D.M., Kraemer, S.B., Schmitt, H.R.,\& Trippe, M.L. 2010, AJ, 140, 577

Fischer, T.C., et al. 2011 ApJ, 727. 71

Gabel, J.R., et al. 2003, ApJ, 583, 178

Gabel, J.R., et al. 2005, ApJ, 631, 741

Ganguly, R. \& Brotherton, M.S. 2008, ApJ, 672, 102

Gebhardt, K., et al. 2000 ApJ, 539L, 13

George, et al. 1998, ApJS, 114, 73

Gibson, R.R., et al. 2009, ApJ, 692, 758

Hamann, F., et al. 2011, MNRAS, 410, 1957

Hopkins, P.F. \& Elvis, M. 2010, MNRAS, 401, 1

Hopkins, P.F., et al. 2005, ApJ, 630, 705

Iwasawa, K. 2000, MNRAS, 318, 879

Kaspi, S., et al. 2003, ApJ, 574, 643

Khalatyan, A., et al. 2008, MNRAS, 387, 13

Kraemer, S.B., Crenshaw, D.M., \& Gabel, J.R. 2001, ApJ, 557, 30.

Kraemer, S.B., Ho, L.C., Crenshaw, D.M., Shields, J.C., \& Filippenko, A.V. 1999, ApJ, 520, 564

Kraemer, S.B., et al. 2001, ApJ, 551, 671.

Kraemer, S.B., et al. 2002, ApJ, 577, 98.

Kraemer, S.B., et al. 2003, ApJ, 582, 125.

Kraemer, S.B., et al. 2005, ApJ, 633, 693 
Kraemer, S.B. et al. 2006, ApJS, 167, 161

Kraemer, S.B. et al. 2011, submitted to ApJ

Kriss, G.A., Peterson, B.M.; Crenshaw, D. M., \& Zheng, W. 2000, ApJ, 535, 58

Kriss, G.A., et al. 2000, ApJ, 538, 17

Kriss, G.A., et al. 2003, A\&A, 403, 473

Kriss, G.A., et al. 2011, A\&A, 534, 41

Krolik, J.H. \& Kriss, G.A. 2001, ApJ, 561, 684

Krongold, Y., Nicastro, F., Brickhouse, N.S., Elvis, M., \& Mathur, S. 2005, ApJ, 622, 842

Krongold, Y., et al. 2010, ApJ, 724, 203

Lobban, A.P., et al. 2011, MNRAS, 414, 1995

Mathur, S., Elvis, M., \& Wilkes, B.J. 1999, ApJ, 519, 605

Matsumoto, C., Leighly, K.M., \& Marshall, H.L. 2004, ApJ, 603, 456

McLure, R.S. \& Dunlop, J.S. 2004 MNRAS, 352, 1390

Moran, E.C., et al. 2005, AJ, 129, 2108

Netzer, H. 2009. ApJ, 695, 793

Netzer, H., et al. 2002, ApJ, 571, 256

Netzer, H., et al. 2003, ApJ, 599, 933.

Nicastro, F., Fiore, F., Perola, G., \& Elvis, M. 1999, ApJ, 512, 184

Peterson, B.M. 1997, An Introduction to Active Galactic Nuclei (Cambridge, UK: Cambridge University Press).

Peterson, B.M., et al. 2004, ApJ, 613, 682

Peterson, B.M., et al. 2005, ApJ, 632, 799

Peterson, B.M., et al. 2006, ApJ, 641, 638

Rafter, S.E., Crenshaw, D.M., \& Wiita, P.J. 2009, AJ, 137, 42

Reynolds, C.S. 1997, MNRAS, 286, 513

Scannapieco, E. \& Oh, S.P. 2004, ApJ, 608, 62

Scott, J.E., et al. 2004, ApJS, 152, 1

Scott, J.E., et al. 2005, ApJ, 634, 193

Scott, J.E., et al. 2009, ApJ, 694, 438 
Shih, D.C., Iwasawa, K., \& Fabian, A.C. 2003, MNRAS, 341, 973

Smith, R.A.N., Page, M.J., \& Branduardi-Raymont, G. 2008, A\&A, 490, 103

Steenbrugge, K. C., et al. 2005, A\&A, 434, 569

Steenbrugge, K.C., Fenovcik, M., Kaastra, J.S., Costantini, E., \& Verbunt, F. 2009, A\&A, 496, 107

Tombesi, F., et al. 2010, A\&A, 521, 57

Turner, T.J., Kraemer, S.B., George, I.M., Reeves, J.N., \& Crenshaw, D.M. 2005, ApJ, 618, 155

Turner, T.J., Miller, L., Kraemer, S.B., \& Reeves, J.N. 2011, ApJ, 733, 48

Turner, T.J., Reeves, J.N., Kraemer, S.B., \& Miller, L. 2008, A\&A, 483, 161

Vestergaard, M. \& Peterson, B.M. 2006, ApJ, 641, 689

Weymann, R.J., Carswell, R.F., \& Smith, M.G. 1981, ARA\&A, 19, 41

Weymann, R.J., Morris, S.K., Gray, M.E., \& Hutchings, J.B. 1997, ApJ, 483, 717

Winter, L.M. 2010, ApJ, 725, 126 
Table 1. AGN Fundamental Parameters

\begin{tabular}{lrcccccc}
\hline \hline \multicolumn{1}{c}{ Name } & $\begin{array}{c}r_{H \beta} \\
(\text { ltday })\end{array}$ & $\begin{array}{c}r_{C I V} \\
(\text { ltday })\end{array}$ & $\begin{array}{c}\log \left(L_{b o l}\right) \\
\left(\mathrm{ergs} \mathrm{s}^{-1}\right)\end{array}$ & $\begin{array}{c}\log \left(M_{B H}\right) \\
\left(\mathrm{M}_{\odot}\right)\end{array}$ & $L_{b o l} / L_{E d d}$ & $\begin{array}{c}\left(\dot{M}_{a c c}\right) \\
\left(\mathrm{M}_{\odot} \mathrm{yr}^{-1}\right)\end{array}$ & Reference \\
\hline NGC 3516 & 11.7 & & 44.16 & 7.50 & 0.036 & 0.026 & 1 \\
NGC 3783 & 10.2 & \multirow{2}{*}{3.8} & 44.25 & 7.47 & 0.047 & 0.032 & 2 \\
NGC 4051 & 1.9 & & 42.81 & 6.24 & 0.030 & 0.001 & 1 \\
NGC 4151 & 6.6 & & 43.87 & 7.66 & 0.013 & 0.013 & 3 \\
NGC 4395 & & \multirow{2}{*}{0.04} & 40.77 & 5.56 & 0.001 & $1.06 \mathrm{E}-5$ & 4 \\
Mrk 279 & 12.5 & & 44.87 & 7.54 & 0.169 & 0.134 & 2 \\
NGC 5548 & 12.4 & \multirow{2}{*}{8.3} & 43.90 & 7.64 & 0.014 & 0.014 & 1 \\
Mrk 509 & 79.6 & & 45.27 & 8.16 & 0.104 & 0.336 & 2 \\
Akn 564 & 17.9 & & 44.61 & 6.42 & 1.242 & 0.074 & 5 \\
NGC 7469 & 4.5 & 2.5 & 44.71 & 7.09 & 0.335 & 0.093 & 2 \\
\hline
\end{tabular}

References. - (1) Denney et al. 2010, (2) Peterson et al. 2004, (3) Bentz et al. 2006; Peterson et al. 2004, (4) Peterson et al. 2005, 2006, (5) Botte et al. 2004. For $r_{C I V}$, the references are Peterson et al. (2004, 2005).

Table 2. AGN Feedback Parameters

\begin{tabular}{ccccc}
\hline \hline Name & $\begin{array}{c}\dot{M}_{\text {out }} \\
\left(\mathrm{M}_{\odot} \mathrm{yr}^{-1}\right)\end{array}$ & $\begin{array}{c}\log \left(\mathrm{L}_{K E}\right) \\
\left(\mathrm{ergs} \mathrm{s}^{-1}\right)\end{array}$ & $\dot{M}_{\text {out }} / \dot{M}_{\text {acc }}$ & $\mathrm{L}_{K E} / \mathrm{L}_{\text {bol }}$ \\
\hline NGC 3516 & $3.8 \mathrm{E}+00-7.7 \mathrm{E}+00$ & $41.73-42.54$ & $150-300$ & $3.7 \mathrm{E}-03-2.4 \mathrm{E}-02$ \\
NGC 3783 & $5.2 \mathrm{E}+00-3.0 \mathrm{E}+01$ & $42.45-42.88$ & $160-940$ & $1.6 \mathrm{E}-02-4.3 \mathrm{E}-02$ \\
NGC 4051 & $1.4 \mathrm{E}-02-1.3 \mathrm{E}-01$ & $40.93-41.93$ & $12-110$ & $1.3 \mathrm{E}-02-1.3 \mathrm{E}-01$ \\
NGC 4151 & $3.2 \mathrm{E}-01-6.7 \mathrm{E}-01$ & $40.40-41.18$ & $24-50$ & $3.4 \mathrm{E}-04-2.0 \mathrm{E}-03$ \\
NGC 4395 & $2.0 \mathrm{E}-07-1.4 \mathrm{E}-04$ & $33.91-36.74$ & $0.02-13$ & $1.4 \mathrm{E}-07-9.4 \mathrm{E}-05$ \\
NGC 5548 & $>7.9 \mathrm{E}-01$ & $>41.26$ & $>55$ & $>2.3 \mathrm{E}-03$ \\
NGC 7469 & $<5.7 \mathrm{E}+00$ & $<42.07$ & $<61$ & $<2.3 \mathrm{E}-03$ \\
\hline
\end{tabular}


Table 3. Feedback Parameters for Individual Kinematic Components

\begin{tabular}{|c|c|c|c|c|c|c|c|c|c|c|c|c|}
\hline Comp. & $\begin{array}{c}v_{r} \\
\left(\mathrm{~km} \mathrm{~s}^{-1}\right)\end{array}$ & $\begin{array}{c}\text { FWHM } \\
\left(\mathrm{km} \mathrm{s}^{-1}\right)\end{array}$ & $\log (\mathrm{U})$ & $\begin{array}{c}\log \left(\mathrm{N}_{H}\right) \\
\left(\mathrm{cm}^{-2}\right)\end{array}$ & $\begin{array}{c}\log \left(r_{C I V}\right) \\
(\mathrm{cm})\end{array}$ & $\begin{array}{c}\log \left(r_{>\Delta r}\right) \\
\quad(\mathrm{cm})\end{array}$ & $\begin{array}{c}\log \left(\mathrm{r}_{\text {min }}\right) \\
\quad(\mathrm{cm})\end{array}$ & $\begin{array}{c}\log \left(\mathrm{r}_{\max }\right) \\
(\mathrm{cm})\end{array}$ & $\begin{array}{c}\dot{M}_{\text {out } \min } \\
\left(\mathrm{M}_{\odot} \mathrm{yr}^{-1}\right)\end{array}$ & $\begin{array}{c}\dot{M}_{\text {out } \max } \\
\left(\mathrm{M}_{\odot} \mathrm{yr}^{-1}\right)\end{array}$ & $\begin{array}{l}L_{K E \min } \\
\left(\mathrm{ergs} \mathrm{s}^{-1}\right)\end{array}$ & $\begin{array}{l}L_{K E \max } \\
\left(\operatorname{ergs~s}^{-1}\right)\end{array}$ \\
\hline \multicolumn{13}{|c|}{ NGC 3516 UV Absorption } \\
\hline $1 \mathrm{a}$ & -376 & 70 & -0.34 & 21.38 & 16.00 & 21.06 & 19.68 & & $1.01 \mathrm{E}+00$ & & $4.48 \mathrm{E}+40$ & \\
\hline $1 b$ & -376 & 70 & -2.35 & 18.85 & 16.00 & 25.60 & 19.68 & & $2.95 \mathrm{E}-03$ & & $1.31 \mathrm{E}+38$ & \\
\hline 2 & -183 & 44 & -0.39 & 21.59 & 16.00 & 20.90 & 19.28 & & $3.18 \mathrm{E}-01$ & & $3.34 \mathrm{E}+39$ & \\
\hline $3+4$ & -36 & 31 & -0.29 & 21.15 & 16.00 & 21.24 & 17.19 & 18.08 & $1.83 \mathrm{E}-04$ & $1.42 \mathrm{E}-03$ & $7.43 \mathrm{E}+34$ & $5.76 \mathrm{E}+35$ \\
\hline 5 & -1372 & 271 & 0.81 & 19.18 & 16.00 & 22.11 & 19.68 & & $2.30 \mathrm{E}-02$ & & $1.36 \mathrm{E}+40$ & \\
\hline 6 & -994 & 36 & 0.93 & 20.18 & 16.00 & 20.99 & 19.68 & & $1.67 \mathrm{E}-01$ & & $5.18 \mathrm{E}+40$ & \\
\hline 7 & -837 & 99 & 1.03 & 21.30 & 16.00 & 19.77 & 19.68 & & $1.87 \mathrm{E}+00$ & & $4.12 \mathrm{E}+41$ & \\
\hline 8 & -692 & 35 & 1.03 & 20.74 & 16.00 & 20.33 & 19.68 & & $4.26 \mathrm{E}-01$ & & $6.40 \mathrm{E}+40$ & \\
\hline UV $1-8$ & & & & & & & & & $3.82 \mathrm{E}+00$ & $3.82 \mathrm{E}+00$ & $5.41 \mathrm{E}+41$ & $5.90 \mathrm{E}+41$ \\
\hline \multicolumn{13}{|c|}{ NGC 3516 X-ray Absorption } \\
\hline $\mathrm{UV}$ & -300 & & -0.90 & 21.70 & & 21.30 & 17.19 & 18.08 & $5.45 \mathrm{E}-03$ & $4.23 \mathrm{E}-02$ & $1.54 \mathrm{E}+38$ & $1.19 \mathrm{E}+39$ \\
\hline $\mathrm{Hi}$ & -1140 & & 1.60 & 22.20 & & 18.30 & & 18.08 & & $5.08 \mathrm{E}-01$ & & $2.07 \mathrm{E}+41$ \\
\hline Heavy & -1600 & & 1.15 & 23.40 & & 17.55 & & 17.55 & & $3.32 \mathrm{E}+00$ & & $2.67 \mathrm{E}+42$ \\
\hline $\mathrm{Hi}+$ Heavy & & & & & & & & & & $3.83 \mathrm{E}+00$ & & $2.87 \mathrm{E}+42$ \\
\hline $\mathrm{UV}+\mathrm{X}$-ray & & & & & & & & & $3.82 \mathrm{E}+00$ & $7.65 \mathrm{E}+00$ & $5.41 \mathrm{E}+41$ & $3.46 \mathrm{E}+42$ \\
\hline \multicolumn{13}{|c|}{ NGC 3783 UV Absorption } \\
\hline $1 \mathrm{a}$ & -1365 & 193 & -1.60 & 20.60 & 15.99 & 23.19 & 19.94 & 19.94 & $1.11 \mathrm{E}+00$ & $1.11 \mathrm{E}+00$ & $6.47 \mathrm{E}+41$ & $6.47 \mathrm{E}+41$ \\
\hline $1 b$ & -1365 & 193 & -0.40 & 21.10 & 15.99 & 21.49 & 19.94 & 19.94 & $3.50 \mathrm{E}+00$ & $3.50 \mathrm{E}+00$ & $2.05 \mathrm{E}+42$ & $2.05 \mathrm{E}+42$ \\
\hline 2 & -548 & 170 & -0.45 & 20.40 & 15.99 & 22.24 & 15.99 & 19.90 & $3.17 \mathrm{E}-05$ & $2.56 \mathrm{E}-01$ & $2.99 \mathrm{E}+36$ & $2.41 \mathrm{E}+40$ \\
\hline 3 & -724 & 280 & -0.50 & 21.10 & 15.99 & 21.59 & 15.99 & 20.20 & $2.10 \mathrm{E}-04$ & $3.38 \mathrm{E}+00$ & $3.95 \mathrm{E}+37$ & $5.56 \mathrm{E}+41$ \\
\hline UV 1-3 & & & & & & & & & $4.61 \mathrm{E}+00$ & $8.25 \mathrm{E}+00$ & $2.69 \mathrm{E}+42$ & $3.27 \mathrm{E}+42$ \\
\hline \multicolumn{13}{|c|}{ NGC 3783 X-ray Absorption } \\
\hline XLI & -800 & & -0.50 & 21.90 & & 20.79 & 19.00 & 20.20 & $1.49 \mathrm{E}+00$ & $1.54 \mathrm{E}+01$ & $2.99 \mathrm{E}+41$ & $3.08 \mathrm{E}+42$ \\
\hline XMI & -800 & & 0.80 & 22.00 & & 19.39 & 18.30 & 19.39 & $3.74 \mathrm{E}-01$ & $4.57 \mathrm{E}+00$ & $7.50 \mathrm{E}+40$ & $9.18 \mathrm{E}+41$ \\
\hline XHI & -800 & & 1.30 & 22.30 & & 18.59 & 17.70 & 18.59 & $1.87 \mathrm{E}-01$ & $1.45 \mathrm{E}+00$ & $3.76 \mathrm{E}+40$ & $2.90 \mathrm{E}+41$ \\
\hline $\mathrm{XMI}+\mathrm{XHI}$ & & & & & & & & & $5.61 \mathrm{E}-01$ & $2.14 \mathrm{E}+01$ & $1.13 \mathrm{E}+41$ & $4.29 \mathrm{E}+42$ \\
\hline $\mathrm{UV}+\mathrm{X}$-ray & & & & & & & & & $5.20 \mathrm{E}+00$ & $2.96 \mathrm{E}+01$ & $2.81 \mathrm{E}+42$ & $7.56 \mathrm{E}+42$ \\
\hline \multicolumn{13}{|c|}{ NGC 4051 UV Absorption } \\
\hline 1 & -647 & 40 & & & 15.21 & & & & & & & \\
\hline 2 & -505 & 165 & -0.72 & 20.17 & 15.21 & 21.30 & 16.70 & & $8.76 \mathrm{E}-05$ & & $7.01 \mathrm{E}+36$ & \\
\hline 3 & -430 & 63 & & & 15.21 & & & & & & & \\
\hline
\end{tabular}


Table 3-Continued

\begin{tabular}{|c|c|c|c|c|c|c|c|c|c|c|c|c|}
\hline Comp. & $\begin{array}{c}v_{r} \\
\left(\mathrm{~km} \mathrm{~s}^{-1}\right)\end{array}$ & $\begin{array}{c}\text { FWHM } \\
\left(\mathrm{km} \mathrm{s}^{-1}\right)\end{array}$ & $\log (\mathrm{U})$ & $\begin{array}{c}\log \left(\mathrm{N}_{H}\right) \\
\left(\mathrm{cm}^{-2}\right)\end{array}$ & $\begin{array}{c}\log \left(r_{C I V}\right) \\
(\mathrm{cm})\end{array}$ & $\begin{array}{c}\log \left(r_{>\Delta r}\right) \\
\quad(\mathrm{cm})\end{array}$ & $\begin{array}{c}\log \left(\mathrm{r}_{\text {min }}\right) \\
(\mathrm{cm})\end{array}$ & $\begin{array}{c}\log \left(\mathrm{r}_{\max }\right) \\
(\mathrm{cm})\end{array}$ & $\begin{array}{c}\dot{M}_{\text {out min }} \\
\left(\mathrm{M}_{\odot} \mathrm{yr}^{-1}\right)\end{array}$ & $\begin{array}{c}\dot{M}_{\text {out } \max } \\
\left(\mathrm{M}_{\odot} \mathrm{yr}^{-1}\right)\end{array}$ & $\begin{array}{l}L_{K E \min } \\
\left(\mathrm{ergs} \mathrm{s}^{-1}\right)\end{array}$ & $\begin{array}{l}L_{K E \max } \\
\left(\mathrm{ergs} \mathrm{s}^{-1}\right)\end{array}$ \\
\hline 4 & -337 & 52 & & & 15.21 & & & & & & & \\
\hline 5 & -268 & 133 & -0.68 & 20.34 & 15.21 & 21.09 & & 17.48 & & $4.14 \mathrm{E}-04$ & & $9.33 \mathrm{E}+36$ \\
\hline 6 & -158 & 45 & & & 15.21 & & & & & & & \\
\hline 7 & -107 & 64 & -0.80 & 20.18 & 15.21 & 21.37 & 17.95 & & $3.38 \mathrm{E}-04$ & & $1.21 \mathrm{E}+36$ & \\
\hline 8 & -48 & 84 & & & 15.21 & & & & & & & \\
\hline 9 & 30 & 23 & & & 15.21 & & & & & & & \\
\hline UV 2 & & & & & & & & & $8.76 \mathrm{E}-05$ & $8.76 \mathrm{E}-05$ & $7.01 \mathrm{E}+36$ & $7.01 \mathrm{E}+36$ \\
\hline \multicolumn{13}{|c|}{ NGC 4051 X-ray Absorption } \\
\hline 1 & -210 & & -1.43 & 20.08 & & 22.10 & 17.95 & & $5.27 \mathrm{E}-04$ & & $7.28 \mathrm{E}+36$ & \\
\hline 2 & -200 & & -0.63 & 20.46 & & 20.92 & & 17.48 & & $4.08 \mathrm{E}-04$ & & $5.11 \mathrm{E}+36$ \\
\hline 3 & -580 & & 0.82 & 20.90 & & 19.03 & 16.70 & & $5.40 \mathrm{E}-04$ & & $5.70 \mathrm{E}+37$ & \\
\hline 4 & -4670 & & 1.69 & 22.30 & & 16.76 & 16.85 & 16.76 & $1.25 \mathrm{E}-02$ & $1.25 \mathrm{E}-01$ & $8.54 \mathrm{E}+40$ & $8.54 \mathrm{E}+41$ \\
\hline X-ray & & & & & & & & & $1.35 \mathrm{E}-02$ & $1.26 \mathrm{E}-01$ & $8.55 \mathrm{E}+40$ & $8.54 \mathrm{E}+41$ \\
\hline $\mathrm{UV}+\mathrm{X}-\mathrm{ray}$ & & & & & & & & & $1.36 \mathrm{E}-02$ & $1.26 \mathrm{E}-01$ & $9.55 \mathrm{E}+40$ & $8.54 \mathrm{E}+41$ \\
\hline \multicolumn{13}{|c|}{ NGC 4151 UV Absorption } \\
\hline $\bar{A}$ & -1588 & 36 & -2.92 & 18.10 & 15.76 & 26.63 & 21.32 & 21.32 & $9.77 \mathrm{E}-02$ & $9.77 \mathrm{E}-02$ & $7.73 \mathrm{E}+40$ & $7.73 \mathrm{E}+40$ \\
\hline $\mathrm{C}$ & -858 & 27 & -2.92 & 18.00 & 15.76 & 26.73 & 21.81 & 21.81 & $1.30 \mathrm{E}-01$ & $1.30 \mathrm{E}-01$ & $2.99 \mathrm{E}+40$ & $2.99 \mathrm{E}+40$ \\
\hline $\mathrm{D}+\mathrm{Ea}$ & -491 & 435 & -0.39 & 22.93 & 15.76 & 19.27 & 17.49 & 17.49 & $3.02 \mathrm{E}-01$ & $3.02 \mathrm{E}-01$ & $2.29 \mathrm{E}+40$ & $2.29 \mathrm{E}+40$ \\
\hline $\mathrm{D}+\mathrm{Eb}$ & -491 & 435 & -1.67 & 20.80 & 15.76 & 22.68 & 17.49 & 17.49 & $2.24 \mathrm{E}-03$ & $2.24 \mathrm{E}-03$ & $1.69 \mathrm{E}+38$ & $1.69 \mathrm{E}+38$ \\
\hline $\mathrm{D}+\mathrm{Ec}$ & -491 & 435 & -1.08 & 21.60 & 15.76 & 21.29 & 17.49 & 17.49 & $1.41 \mathrm{E}-02$ & $1.41 \mathrm{E}-02$ & $1.07 \mathrm{E}+39$ & $1.07 \mathrm{E}+39$ \\
\hline $\mathrm{D}+\mathrm{Ed}$ & -491 & 435 & -3.35 & 19.50 & 15.76 & 25.66 & 17.49 & 17.49 & $1.12 \mathrm{E}-04$ & $1.12 \mathrm{E}-04$ & $8.49 \mathrm{E}+36$ & $8.49 \mathrm{E}+36$ \\
\hline$D^{\prime}$ & -1680 & 940 & 0.31 & 20.00 & 15.76 & 21.50 & 17.49 & 18.49 & $1.21 \mathrm{E}-03$ & $1.21 \mathrm{E}-02$ & $1.08 \mathrm{E}+39$ & $1.08 \mathrm{E}+40$ \\
\hline E'a & -215 & 59 & -1.74 & 20.60 & 15.76 & 22.95 & 18.27 & 18.27 & $3.73 \mathrm{E}-03$ & $3.73 \mathrm{E}-03$ & $5.41 \mathrm{E}+37$ & $5.41 \mathrm{E}+37$ \\
\hline E'b & -215 & 59 & -3.64 & 19.00 & 15.76 & 26.45 & 18.27 & 18.27 & $9.37 \mathrm{E}-05$ & $9.37 \mathrm{E}-05$ & $1.36 \mathrm{E}+36$ & $1.36 \mathrm{E}+36$ \\
\hline UV & & & & & & & & & $3.24 \mathrm{E}-01$ & $5.62 \mathrm{E}-01$ & $2.52 \mathrm{E}+40$ & $1.42 \mathrm{E}+41$ \\
\hline \multicolumn{13}{|c|}{ NGC 4151 X-ray Absorption } \\
\hline Xhigh & -491 & & 1.05 & 22.50 & & 18.26 & & 17.49 & & $1.12 \mathrm{E}-01$ & & $8.49 \mathrm{E}+39$ \\
\hline $\mathrm{UV}+\mathrm{X}$-ray & & & & & & & & & $3.20 \mathrm{E}-01$ & $6.70 \mathrm{E}-01$ & $2.52 \mathrm{E}+40$ & $1.51 \mathrm{E}+41$ \\
\hline \multicolumn{13}{|c|}{ NGC 4395 UV Absorption } \\
\hline $1(\mathrm{~B})$ & -840 & & -1.70 & 19.00 & 14.02 & 21.41 & 14.02 & 16.85 & $2.04 \mathrm{E}-08$ & $1.39 \mathrm{E}-05$ & $4.51 \mathrm{E}+33$ & $3.08 \mathrm{E}+36$ \\
\hline $2(\mathrm{Ah})$ & -250 & & -0.70 & 20.48 & 14.02 & 18.93 & 14.02 & 16.85 & $1.83 \mathrm{E}-07$ & $1.25 \mathrm{E}-04$ & $3.59 \mathrm{E}+33$ & $2.45 \mathrm{E}+36$ \\
\hline UV & & & & & & & & & $2.03 \mathrm{E}-07$ & $1.39 \mathrm{E}-04$ & $8.10 \mathrm{E}+33$ & $5.53 \mathrm{E}+36$ \\
\hline
\end{tabular}


Table 3 - Continued

\begin{tabular}{|c|c|c|c|c|c|c|c|c|c|c|c|c|}
\hline Comp. & $\begin{array}{c}v_{r} \\
\left(\mathrm{~km} \mathrm{~s}^{-1}\right)\end{array}$ & $\begin{array}{c}\text { FWHM } \\
\left(\mathrm{km} \mathrm{s}^{-1}\right)\end{array}$ & $\log (U)$ & $\begin{array}{c}\log \left(\mathrm{N}_{H}\right) \\
\left(\mathrm{cm}^{-2}\right)\end{array}$ & $\begin{array}{c}\log \left(r_{C I V}\right) \\
(\mathrm{cm})\end{array}$ & $\begin{array}{c}\log \left(r_{>\Delta r}\right) \\
\quad(\mathrm{cm})\end{array}$ & $\begin{array}{c}\log \left(\mathrm{r}_{\min }\right) \\
(\mathrm{cm})\end{array}$ & $\begin{array}{c}\log \left(\mathrm{r}_{\max }\right) \\
(\mathrm{cm})\end{array}$ & $\begin{array}{c}\dot{M}_{\text {out min }} \\
\left(\mathrm{M}_{\odot} \mathrm{yr}^{-1}\right)\end{array}$ & $\begin{array}{c}\dot{M}_{\text {out } \max } \\
\left(\mathrm{M}_{\odot} \mathrm{yr}^{-1}\right)\end{array}$ & $\begin{array}{c}L_{K E \min } \\
\left(\mathrm{ergs} \mathrm{s}^{-1}\right)\end{array}$ & $\begin{array}{l}L_{K E \max } \\
\left(\operatorname{ergs~s}^{-1}\right)\end{array}$ \\
\hline \multicolumn{13}{|c|}{ NGC 4395 X-ray Absorption } \\
\hline Constant & & & 0.80 & 22.39 & & 15.52 & & 15.52 & & & & \\
\hline Variable & & & 1.18 & 22.90 & & 14.63 & & 14.63 & & & & \\
\hline \multicolumn{13}{|l|}{ X-ray } \\
\hline \multicolumn{13}{|l|}{$\mathrm{UV}+\mathrm{X}$-ray } \\
\hline \multicolumn{13}{|c|}{ Mrk 279 UV Absorption } \\
\hline 1 & 90 & & & & & & & & & & & \\
\hline 2 & -265 & & -1.00 & 18.90 & 16.03 & 24.91 & & & & & & \\
\hline 3 & -385 & & & & & & & & & & & \\
\hline 4 & -450 & & & & & & & & & & & \\
\hline 5 & -540 & & & & & & & & & & & \\
\hline \multicolumn{13}{|l|}{ UV } \\
\hline \multicolumn{13}{|c|}{ MRK 279 X-ray Absorption } \\
\hline 1 & -200 & & -0.80 & 19.85 & & 23.76 & & & & & & \\
\hline 2 & -370 & & 1.10 & 20.43 & & 21.28 & & & & & & \\
\hline \multicolumn{13}{|l|}{ X-ray } \\
\hline \multicolumn{13}{|c|}{ NGC 5548 UV Absorption } \\
\hline 1 & -1040 & 220 & -0.59 & 19.96 & 16.33 & 22.47 & 20.33 & & $4.75 \mathrm{E}-01$ & & $1.61 \mathrm{E}+41$ & \\
\hline 2 & -670 & 40 & -0.78 & 19.20 & 16.33 & 23.42 & 20.33 & & $5.31 \mathrm{E}-02$ & & $7.48 \mathrm{E}+39$ & \\
\hline 3 & -530 & 160 & -0.15 & 21.77 & 16.33 & 20.22 & & 19.00 & & $7.30 \mathrm{E}-01$ & & $6.43 \mathrm{E}+40$ \\
\hline 4 & -340 & 150 & -0.67 & 20.13 & 16.33 & 22.38 & 20.33 & & $2.29 \mathrm{E}-01$ & & $8.32 \mathrm{E}+39$ & \\
\hline 5 & -170 & 60 & -0.67 & 19.41 & 16.33 & 23.10 & 20.33 & & $2.19 \mathrm{E}-02$ & & $1.98 \mathrm{E}+38$ & \\
\hline UV $1-5$ & & & & & & & & & $7.79 \mathrm{E}-01$ & & $1.77 \mathrm{E}+41$ & \\
\hline \multicolumn{13}{|c|}{ NGC 5548 X-ray Absorption } \\
\hline HV-SHIP & -1040 & & 1.23 & 21.73 & & 18.88 & 17.00 & & $1.31 \mathrm{E}-02$ & & $4.43 \mathrm{E}+39$ & \\
\hline HV-HIP & -1180 & & 0.67 & 21.03 & & 20.14 & 17.00 & & $2.96 \mathrm{E}-03$ & & $1.29 \mathrm{E}+39$ & \\
\hline LV-HIP & -400 & & 0.67 & 21.26 & & 19.91 & & 19.00 & & $1.70 \mathrm{E}-01$ & & $8.55 \mathrm{E}+39$ \\
\hline LV-LIP & -590 & & -0.49 & 20.75 & & 21.58 & & 19.00 & & $7.76 \mathrm{E}-02$ & & $8.48 \mathrm{E}+39$ \\
\hline X-ray & & & & & & & & & $1.60 \mathrm{E}-02$ & & $5.73 \mathrm{E}+39$ & \\
\hline $\mathrm{UV}+\mathrm{X}$-ray & & & & & & & & & $7.95 \mathrm{E}-01$ & & $1.83 \mathrm{E}+41$ & \\
\hline \multicolumn{13}{|c|}{ MRK 509 UV Absorption } \\
\hline 1 & -422 & 28 & -0.82 & 19.01 & 16.84 & 25.02 & & 20.89 & & $7.80 \mathrm{E}-02$ & & $4.35 \mathrm{E}+39$ \\
\hline
\end{tabular}


Table 3-Continued

\begin{tabular}{|c|c|c|c|c|c|c|c|c|c|c|c|c|}
\hline Comp. & $\begin{array}{c}v_{r} \\
\left(\mathrm{~km} \mathrm{~s}^{-1}\right)\end{array}$ & $\begin{array}{c}\text { FWHM } \\
\left(\mathrm{km} \mathrm{s}^{-1}\right)\end{array}$ & $\log (\mathrm{U})$ & $\begin{array}{c}\log \left(\mathrm{N}_{H}\right) \\
\left(\mathrm{cm}^{-2}\right)\end{array}$ & $\begin{array}{c}\log \left(r_{C I V}\right) \\
(\mathrm{cm})\end{array}$ & $\begin{array}{c}\log \left(r_{>\Delta r}\right) \\
\quad(\mathrm{cm})\end{array}$ & $\begin{array}{c}\log \left(\mathrm{r}_{\text {min }}\right) \\
\quad(\mathrm{cm})\end{array}$ & $\begin{array}{c}\log \left(\mathrm{r}_{\max }\right) \\
(\mathrm{cm})\end{array}$ & $\begin{array}{c}\dot{M}_{\text {out min }} \\
\left(\mathrm{M}_{\odot} \mathrm{yr}^{-1}\right)\end{array}$ & $\begin{array}{c}\dot{M}_{\text {out } \max } \\
\left(\mathrm{M}_{\odot} \mathrm{yr}^{-1}\right)\end{array}$ & $\begin{array}{c}L_{K E \min } \\
\left(\mathrm{ergs} \mathrm{s}^{-1}\right)\end{array}$ & $\begin{array}{l}L_{K E \max } \\
\left(\mathrm{ergs} \mathrm{s}^{-1}\right)\end{array}$ \\
\hline 2 & -328 & 49 & -1.31 & 18.92 & 16.84 & 25.60 & & & & & & \\
\hline 3 & -259 & 41 & -1.48 & 18.25 & 16.84 & 26.44 & & & & & & \\
\hline $4^{\prime}$ & -62 & 32 & -1.19 & 18.53 & 16.84 & 25.87 & & & & & & \\
\hline $4 \mathrm{~h}$ & -22 & 52 & -1.02 & 18.97 & 16.84 & 25.26 & & & & & & \\
\hline 41 & -21 & 21 & -1.70 & 19.53 & 16.84 & 25.38 & & & & & & \\
\hline 5 & 34 & 35 & -0.82 & 18.59 & 16.84 & 25.44 & & & & & & \\
\hline 6 & 124 & 29 & -0.78 & 18.79 & 16.84 & 25.20 & & & & & & \\
\hline 7 & 210 & 53 & -0.16 & 19.60 & 16.84 & 23.77 & & & & & & \\
\hline UV & & & & & & & & & & $7.80 \mathrm{E}-02$ & & $4.35 \mathrm{E}+39$ \\
\hline \multicolumn{13}{|c|}{ Mrk 509 X-ray Absorption } \\
\hline 1 & 73 & & -0.44 & 20.27 & & 23.38 & & & & & & \\
\hline 2 & -196 & & 0.76 & 20.73 & & 21.72 & & & & & & \\
\hline 3 & -455 & & 1.65 & 20.78 & & 20.78 & & 18.19 & & $9.94 \mathrm{E}-03$ & & $6.45 \mathrm{E}+38$ \\
\hline X-ray & & & & & & & & & & $9.94 \mathrm{E}-03$ & & $6.45 \mathrm{E}+38$ \\
\hline UV+X-ray & & & & & & & & & & $8.79 \mathrm{E}-02$ & & $5.00 \mathrm{E}+39$ \\
\hline \multicolumn{13}{|c|}{ Akn 564 UV Absorption } \\
\hline 1 & $\begin{array}{l}-190 \\
-19\end{array}$ & 180 & -1.48 & 21.21 & 16.19 & 22.82 & 20.47 & & $2.11 \mathrm{E}+00$ & & $2.39 \mathrm{E}+40$ & \\
\hline \multicolumn{13}{|c|}{ Akn564 X-ray Absorption } \\
\hline 1 & & & -2.36 & 19.95 & & 24.96 & & & & & & \\
\hline 2 & -40 & & -0.63 & 20.38 & & 22.80 & & & & & & \\
\hline 3 & -10 & & 1.06 & 20.78 & & 20.71 & & & & & & \\
\hline \multicolumn{13}{|l|}{ X-ray } \\
\hline \multicolumn{13}{|l|}{ UV+X-ray } \\
\hline \multicolumn{13}{|c|}{ NGC 7469 UV Absorption } \\
\hline 1 & -560 & & 0.00 & 20.00 & 15.81 & 22.65 & & 20.49 & & $4.04 \mathrm{E}-01$ & & $3.98 \mathrm{E}+40$ \\
\hline 2 & -1900 & & -1.10 & 18.60 & 15.81 & 25.15 & & 21.27 & & $3.28 \mathrm{E}-01$ & & $3.71 \mathrm{E}+41$ \\
\hline UV 1-2 & & & & & & & & & & $7.32 \mathrm{E}-01$ & & $4.11 \mathrm{E}+41$ \\
\hline \multicolumn{13}{|c|}{ NGC 7469 X-ray Absorption } \\
\hline 1 & -2300 & & -0.70 & 19.48 & & 23.87 & & 23.87 & & & & \\
\hline 2 & -720 & & 1.23 & 21.30 & & 20.12 & & 20.12 & & $4.41 \mathrm{E}+00$ & & $7.17 \mathrm{E}+41$ \\
\hline 3 & -580 & & 2.06 & 21.46 & & 19.13 & & 19.13 & & $5.25 \mathrm{E}-01$ & & $5.54 \mathrm{E}+40$ \\
\hline X-ray 2-3 & & & & & & & & & & $4.93 \mathrm{E}+00$ & & $7.72 \mathrm{E}+41$ \\
\hline
\end{tabular}


Table 3 - Continued

\begin{tabular}{|c|c|c|c|c|c|c|c|c|c|c|c|c|}
\hline Comp. & $\begin{array}{c}v_{r} \\
\left(\mathrm{~km} \mathrm{~s}^{-1}\right)\end{array}$ & $\begin{array}{c}\text { FWHM } \\
\left(\mathrm{km} \mathrm{s}^{-1}\right)\end{array}$ & $\log (\mathrm{U})$ & $\begin{array}{c}\log \left(\mathrm{N}_{H}\right) \\
\left(\mathrm{cm}^{-2}\right)\end{array}$ & $\begin{array}{c}\log \left(r_{C I V}\right) \\
(\mathrm{cm})\end{array}$ & $\begin{array}{c}\log \left(r_{>\Delta r}\right) \\
\quad(\mathrm{cm})\end{array}$ & $\begin{array}{c}\log \left(\mathrm{r}_{\min }\right) \\
\quad(\mathrm{cm})\end{array}$ & $\begin{array}{c}\log \left(\mathrm{r}_{\max }\right) \\
\quad(\mathrm{cm})\end{array}$ & $\begin{array}{c}\dot{M}_{\text {out } \min } \\
\left(\mathrm{M}_{\odot} \mathrm{yr}^{-1}\right)\end{array}$ & $\begin{array}{c}\dot{M}_{\text {out } \max } \\
\left(\mathrm{M}_{\odot} \mathrm{yr}^{-1}\right)\end{array}$ & $\begin{array}{c}L_{K E \min } \\
\left(\operatorname{ergs~s}^{-1}\right)\end{array}$ & $\begin{array}{c}L_{K E \max } \\
\left(\mathrm{ergs} \mathrm{s}^{-1}\right)\end{array}$ \\
\hline $\mathrm{UV}+\mathrm{X}$-ray & & & & & & & & & & $5.67 \mathrm{E}+00$ & & $1.18 \mathrm{E}+42$ \\
\hline
\end{tabular}


Fig. 1.- FWHM vs. radial-velocity centroid for the UV absorbers.

Fig. 2.- Ionization parameter vs. radial-velocity centroid for the UV (open circles) and X-ray (filled circles) absorbers.

Fig. 3.- Ionization parameter vs. column density $\left(\mathrm{cm}^{-2}\right)$ for the UV (open circles) and X-ray (filled circles) absorbers.

Fig. 4. - Radial location (or limit) vs. radial velocity centroid $\left(\mathrm{km} \mathrm{s}^{-1}\right)$ for the UV (open circles) and X-ray (filled circles) absorbers.

Fig. 5.- Ratio of total mass outflow rate to inferred accretion rate as a function of bolometric luminosity, based on the minimum and maximum values given in Table 2.

Fig. 6.- Ratio of total kinetic luminosity to bolometric luminosity as a function of bolometric luminosity, based on the minimum and maximum values given in Table 2 . 


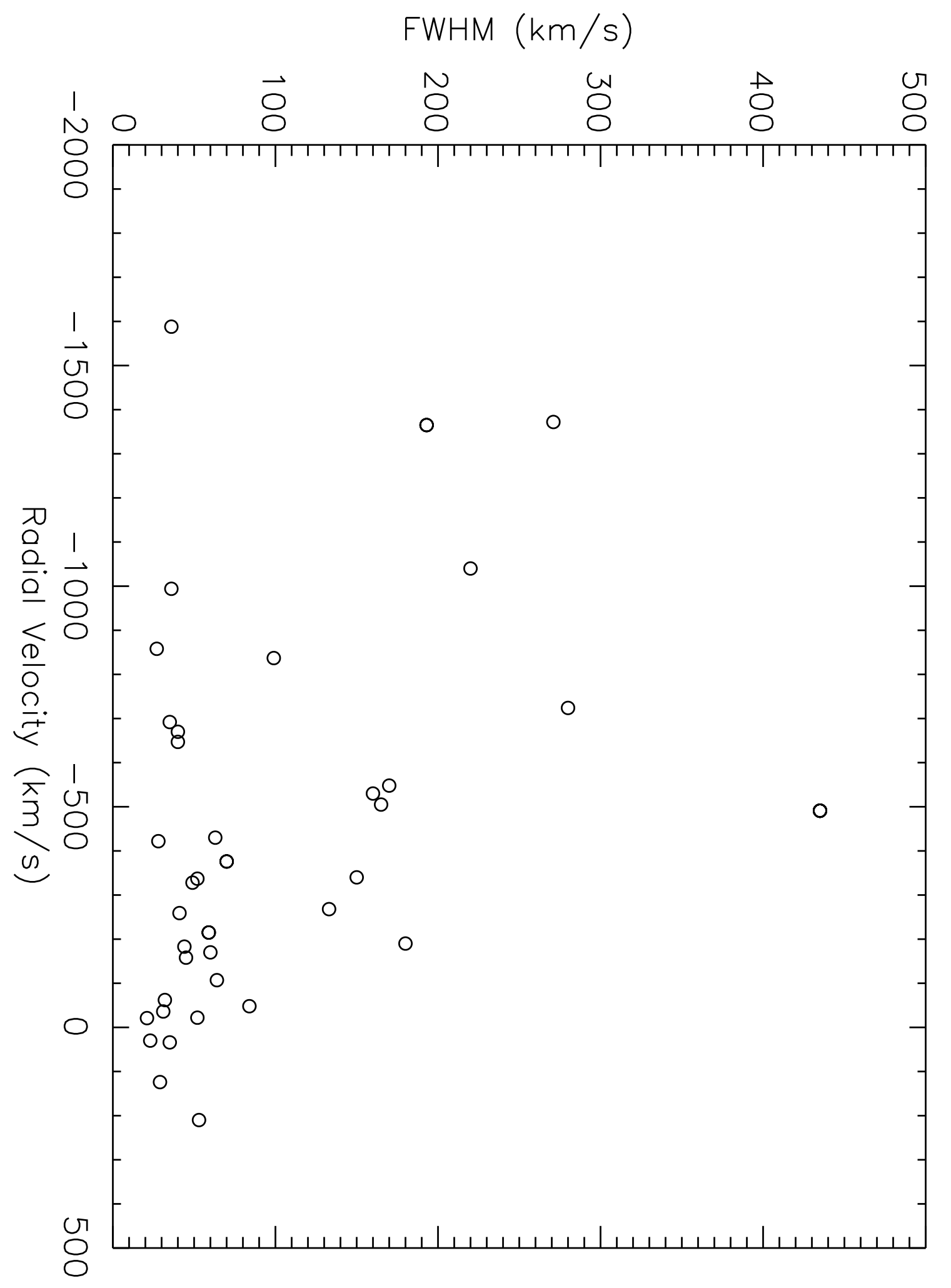




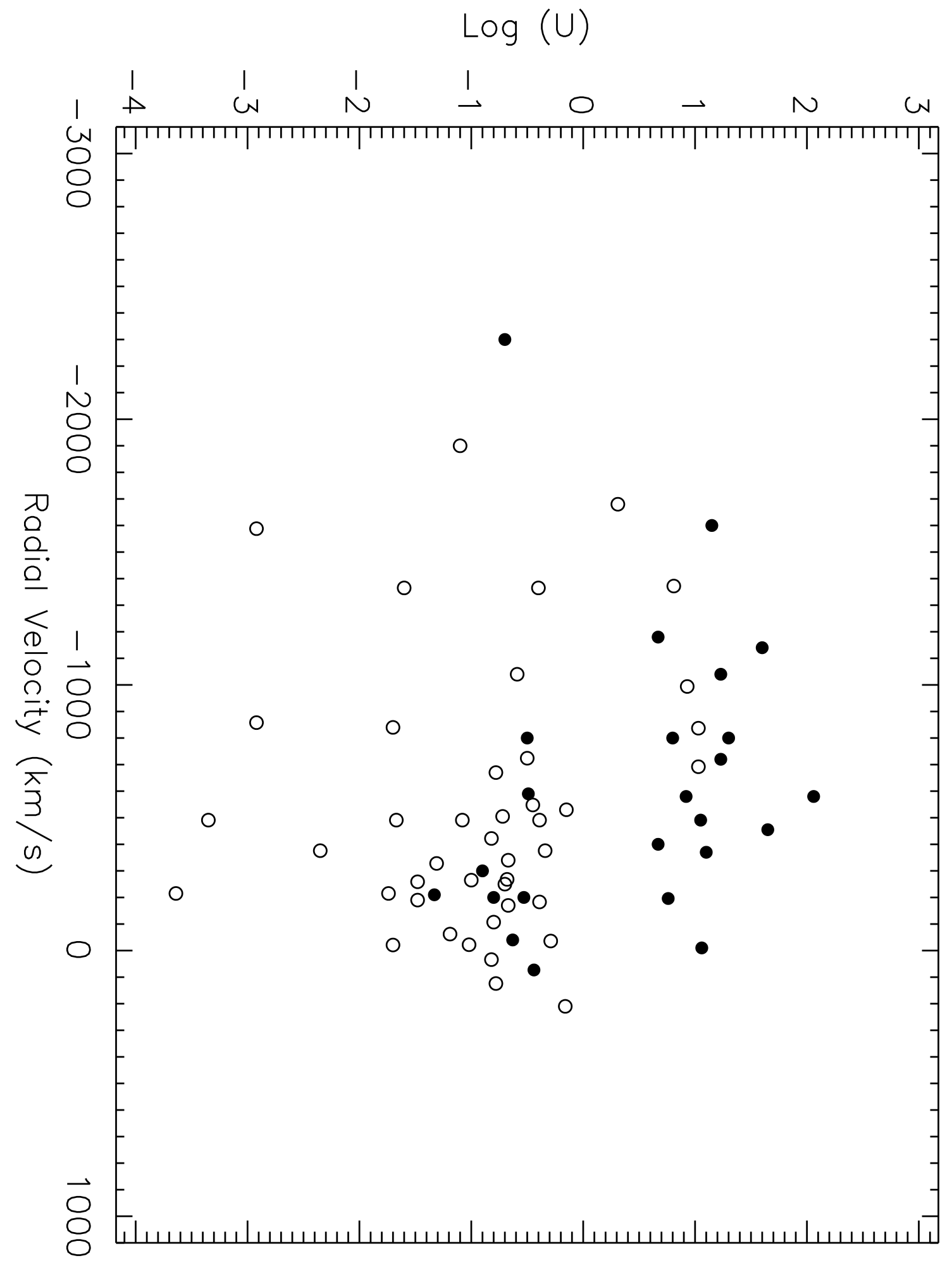




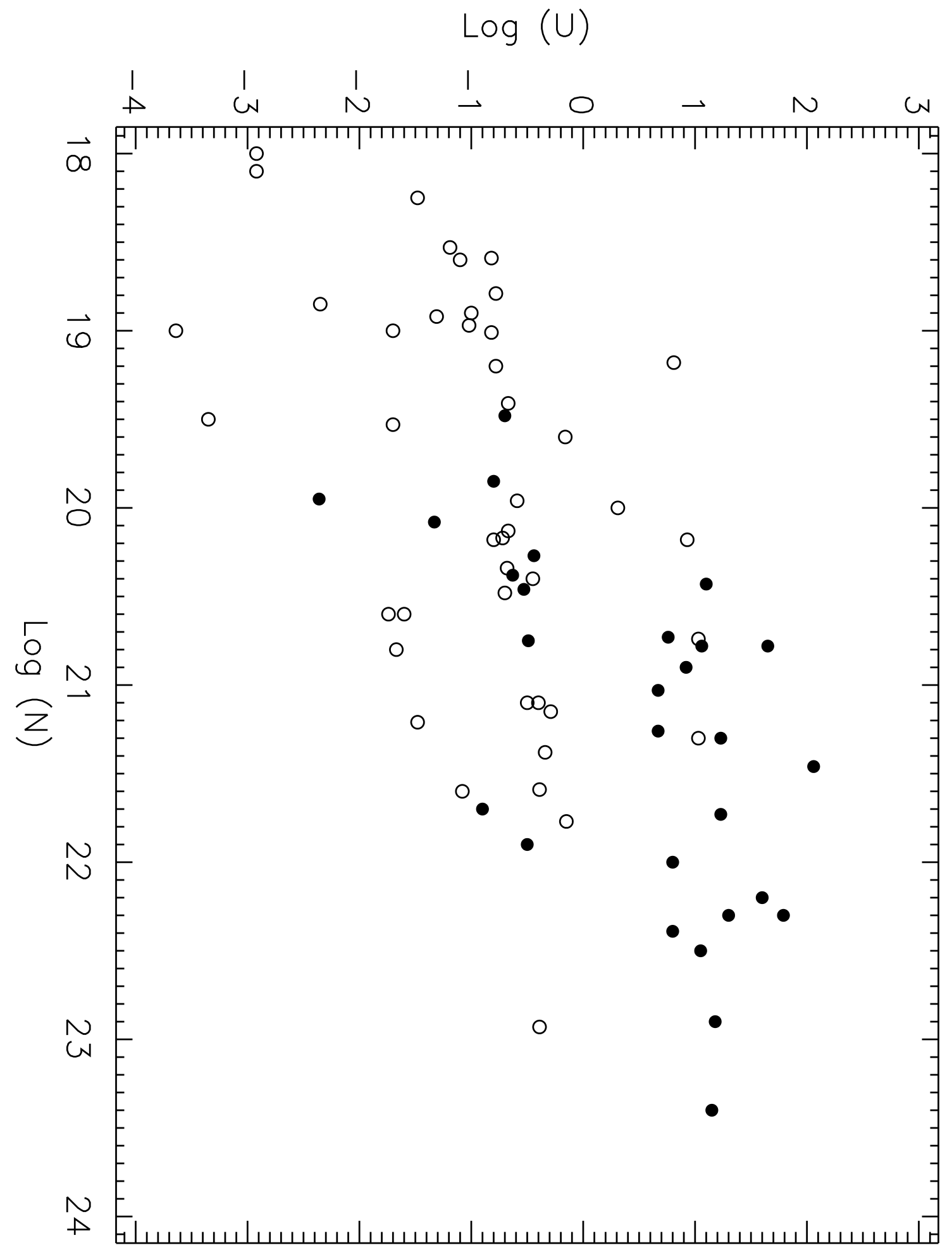




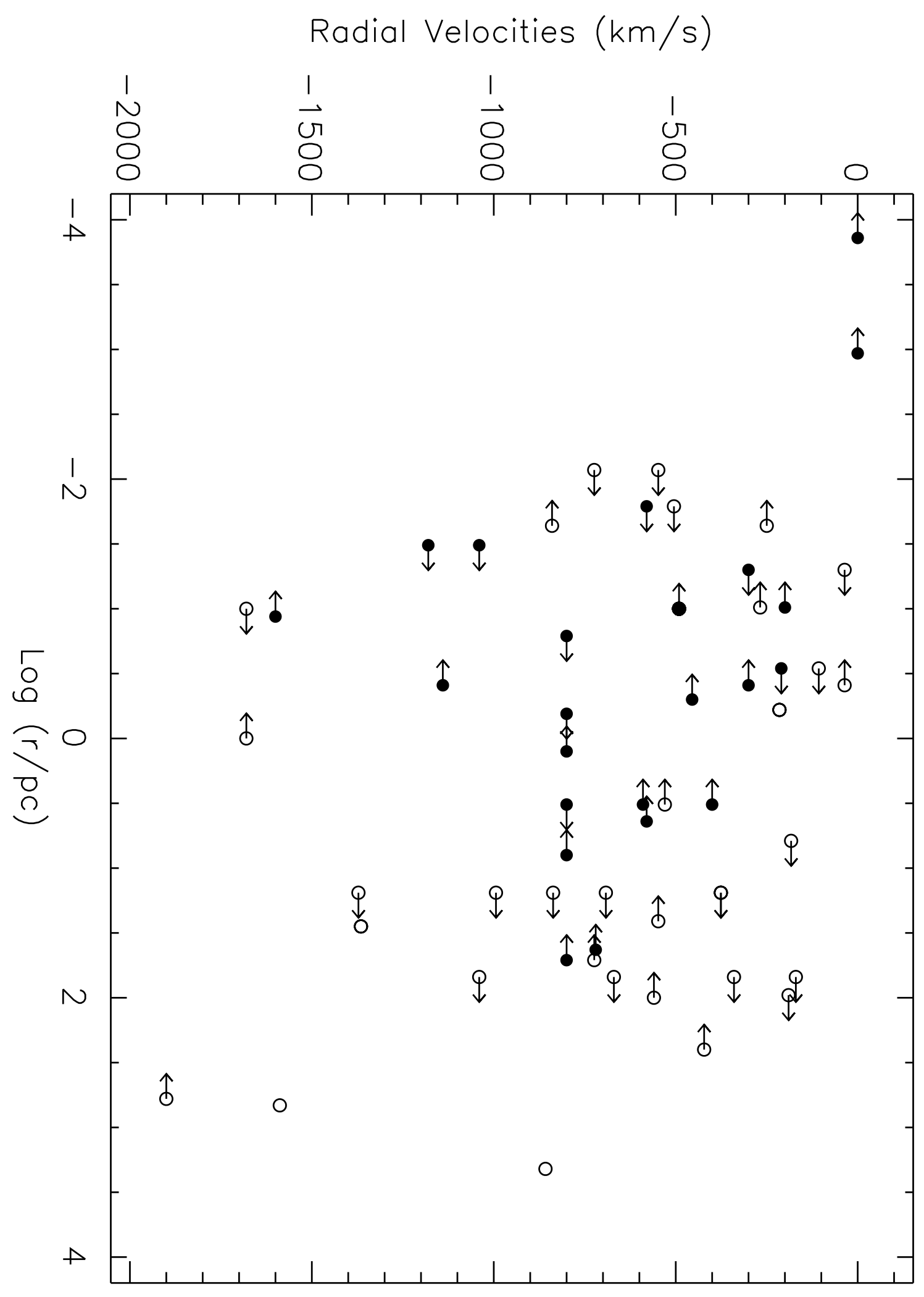




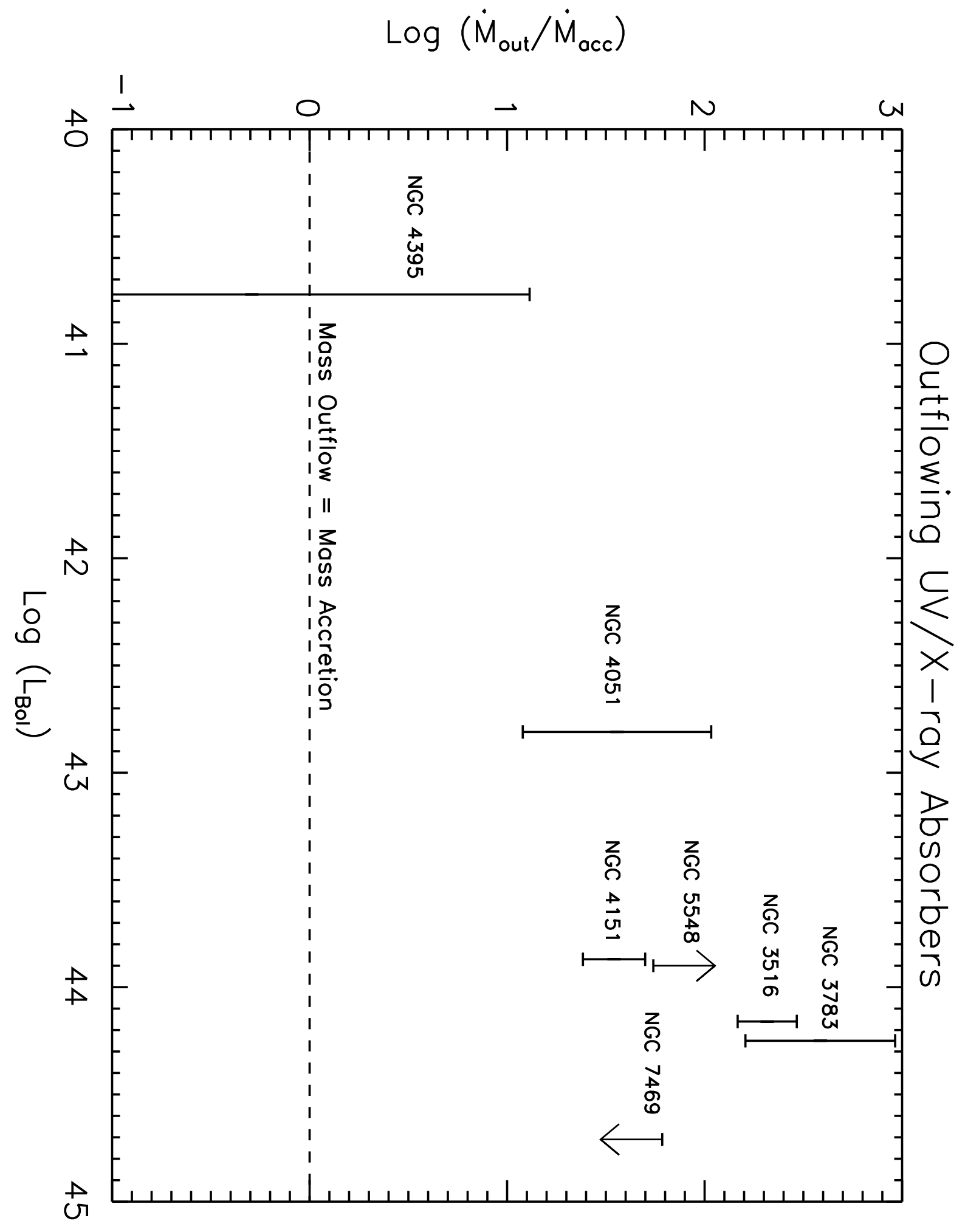




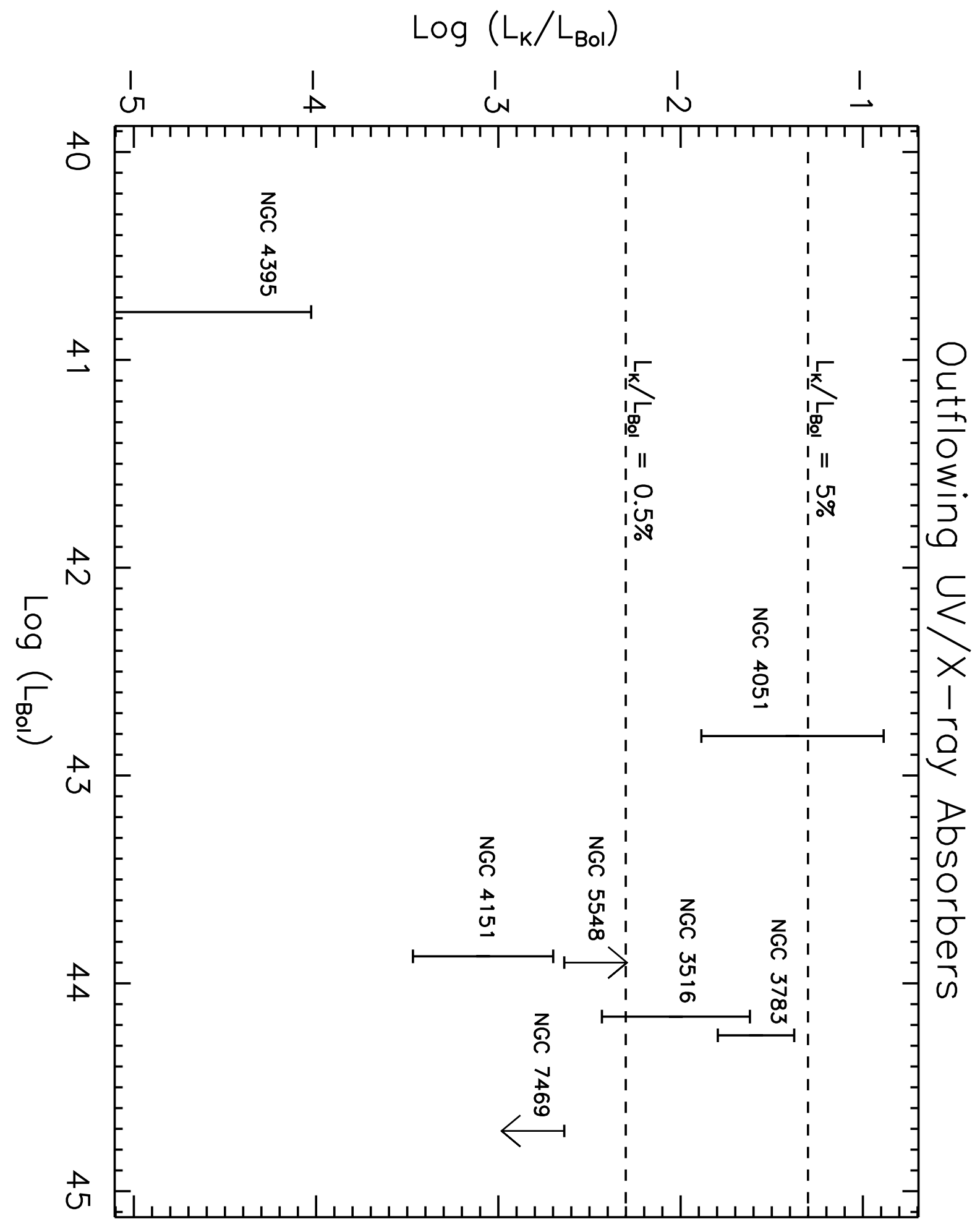

